\title{
Adaptive optics based on machine learning: a review
}

\author{
Youming Guo $\mathrm{G}^{1,2,3}$, Libo Zhong ${ }^{1,2}$, Lei Min ${ }^{1,2}$, Jiaying Wang ${ }^{1,2,3}, \mathrm{Yu} \mathrm{Wu}^{1,2,3}$, \\ Kele Chen ${ }^{1,2,3}$, Kai Wei ${ }^{1,2,3}$ and Changhui Rao ${ }^{1,2,3 *}$
}

\begin{abstract}
Adaptive optics techniques have been developed over the past half century and routinely used in large ground-based telescopes for more than 30 years. Although this technique has already been used in various applications, the basic setup and methods have not changed over the past 40 years. In recent years, with the rapid development of artificial intelligence, adaptive optics will be boosted dramatically. In this paper, the recent advances on almost all aspects of adaptive optics based on machine learning are summarized. The state-of-the-art performance of intelligent adaptive optics are reviewed. The potential advantages and deficiencies of intelligent adaptive optics are also discussed.
\end{abstract}

Keywords: adaptive optics; machine learning; deep learning

Guo YM, Zhong LB, Min L, Wang JY, Wu Y et al. Adaptive optics based on machine learning: a review. Opto-Electron Adv 5, 200082 (2022).

\section{Introduction}

Adaptive optics (AO) is a dynamic wavefront compensation technique widely used in various applications such as ground-based telescopes ${ }^{1,2}$, laser communication ${ }^{3}$, and biological imaging ${ }^{4,5}$ et al. In astronomy, almost all of the ground-based high-resolution imaging telescopes with apertures larger than $1 \mathrm{~m}$ have been equipped with $\mathrm{AO}$ systems (AOS). In microscopy, AO is becoming a valuable tool for high resolution microscopy, providing correction for aberrations introduced by the refractive index structure of specimens ${ }^{6}$. In quantum communication, AO is used to compensate the effects of atmospheric distortion to maximize the quality of the optical link and reduce the turbulence induced loss and noise at the receiver ${ }^{7}$. In 2019, the Air Force Research Laboratory Starfire Optical Range demonstrated that quantum com- munication with $\mathrm{AO}$ can support the quantum communication through-the-air in daylight under conditions representative of space-to-Earth satellite links ${ }^{8}$. In retinal imaging, AO is being used to enhance the ability of optical coherence tomography, fluorescence imaging, and reflectance imaging 9 .

Our laboratory, the key laboratory on AO in Chinese Academy of Sciences is one of the largest teams in the world working on AO which has the capability to develop the complete set of AOS. We apply AO techniques to astronomical telescope ${ }^{10-12}$, inertial confinement fusion ${ }^{13}$, space-to-earth laser communication ${ }^{14}$, retinal imaging ${ }^{15}$ et al. Here brief introduction of some recent developments in the laboratory is presented. In astronomical observation, we did the first on-sky demonstration of piezoelectric adaptive secondary mirror based AOS in

${ }^{1}$ The Key Laboratory on Adaptive Optics, Chinese Academy of Sciences, Chengdu 610209, China; ${ }^{2}$ The Laboratory on Adaptive Optics, Institute of Optics and Electronics, Chinese Academy of Sciences, Chengdu 610209, China; 3University of Chinese Academy of Sciences, Beijing 100049, China.

${ }^{*}$ Correspondence: CH Rao, E-mail: chrao@ioe.ac.cn

Received: 23 November 2020; Accepted: 2 March 2021; Published online: 28 January 2022

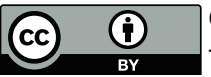

Open Access This article is licensed under a Creative Commons Attribution 4.0 International License.

To view a copy of this license, visit http://creativecommons.org/licenses/by/4.0/.

(c) The Author(s) 2022. Published by Institute of Optics and Electronics, Chinese Academy of Sciences. 
$2016^{16}$. We built and successfully tested the first multiconjugate AO (MCAO) system in China on the 1-m New Vacuum Solar Telescope in $2017^{17}$. We also built one of the largest solar telescopes in the world, the $1.8-\mathrm{m}$ Chinese Large Solar Telescope equipped with a 451-unit AOS and the telescope saw its first light in $2020^{18}$. In free-space laser communication, an experiment of a 5Gbps free-space coherent optical communication system was finished with bit-error rate under $10^{-6}$ after AO compensation ${ }^{19}$. Besides, different kinds of deformable mirrors have been developed and used in different areas ${ }^{20}$.

Although AO has been already successfully implemented in many areas to improve the image resolution or peak energy of lasers, there are still some challenging problems. For example, how to get the wavefront of wavefront sensor less (WFS-less) $\mathrm{AOS}^{21}$ or array telescopes with high speed, how to estimate the wavefront along the line of sight to the scientific target in multi-object AO (MOAO) system ${ }^{22}$ and how to reduce the timedelay error in extreme AOSs ${ }^{23}$, etc. With the development of machine learning, especially the deep learning techniques ${ }^{24}$, some of these complex or inverse problems can be solved. Machine learning is a concept that an algorithm can learn and adapt to new data without human intervention. It is usually divided into two kinds of methods, supervised learning and unsupervised learning. Supervised learning is provided with training data containing not only inputs but also outputs that are also named as labels. The most popular collection of supervised learning algorithms is deep learning which is composed by multiple layers of neural networks. In general, at least three important tasks in AO require such tools including determining the aberration from optically modulated images in WFS, predicting future wavefront with historical multi-source information and reconstructing the high-resolution images from the noisy and blurred ones. These problems are either ill-conditioned or highly nonlinear. Typical traditional algorithms such as least square fit (LSF), singular value decomposition (SVD), or GaussSeidel et al. either have weak fitting capability or require many iterations. On the contrary, deep learning algorithms not only have strong fitting capabilities but also can contain the prior information in the network's structure and weights. These natures can help AO solve the above problems. Besides, the structure of AOS may be also simplified by the powerful algorithms and computation.

Machine learning in AO was investigated as early as
$1990 \mathrm{~s}^{25-27}$. At that time, artificial neural networks (ANN) were considered to be a good alternative for the wavefront sensing of single-aperture and array telescopes in the multiple mirror telescope (MMT) ${ }^{28}$. Experiment has been done on the MMT to demonstrate the advantages of the $\mathrm{ANN}^{29}$. The same technique was used to estimate the aberration of Hubble space telescope and got almost the same result of slow off-line Fourier based phase-retrieval methods ${ }^{30}$. Meanwhile, the prediction of turbulence with ANN was also studied and some simulation results showed potential superiority ${ }^{31}$.

With the fast development of deep learning algorithms, computation power and explosive expansion of data, we have already seen great advancements in computer vision, speech recognition and natural language processing, etc. In recent years, rapidly growing number of researches working on machine learning-assisted intelligent AO (IAO) have been published and more are expected in the near future. In this review, recent advancements on IAO are summarized and future development trends are discussed. This review is organized as follows. Traditional AO is briefly introduced in Section Brief introduction of adaptive optics system, IAO including the intelligent wavefront sensing, intelligent wavefront prediction, intelligent post-processing as well as other applications are described in Section Intelligent adaptive optics, and finally in Section Conclusion \& discussion, we draw the conclusion and discuss the future development of IAO.

\section{Brief introduction of adaptive optics system}

Taking the solar observation as an example, the AOS is basically composed of a deformable mirror (DM), a WFS, a real-time controller (RTC), and a post-processing program, as described in Fig. 1.

\section{Traditional wavefront sensing}

Wavefront sensing is a technique to provide a signal with which the shape of the wavefront can be estimated with sufficient accuracy ${ }^{32}$. Several kinds of WFSs are frequently used in different AOSs including the ShackHartmann WFS ${ }^{33}$ (SHWFS), Pyramid WFS ${ }^{34}$ and curvature $\mathrm{WFS}^{35}$ as shown in Fig. 2. These traditional WFSs build linear relationship between the wavefront and the selected features such as sub-aperture slopes, wavefront curvatures or image intensity differences. On one hand, owing to the simple relationship, wavefront can be reconstructed quickly enough to satisfy the 


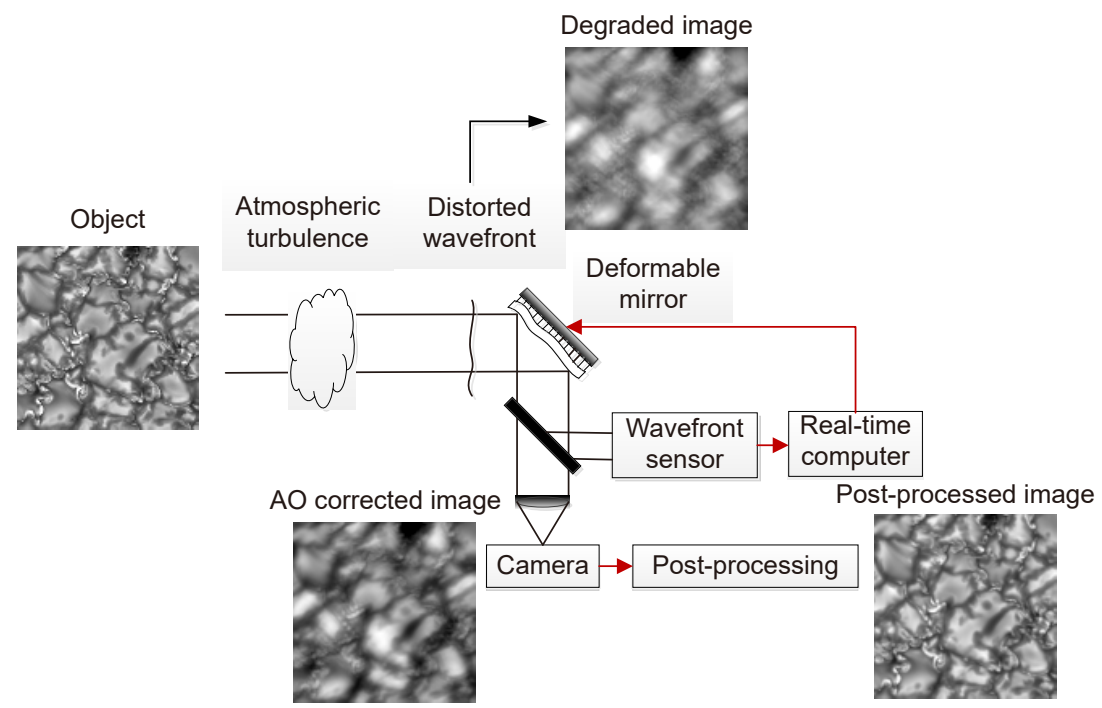

Fig. 1 | Overview of AOS for solar observation. The light waves emitted by the Sun suffer from wavefront distortion when pass through the atmospheric turbulence. The WFS detects the intensity distributions caused by the wavefront distortion and then transfers them to the RTC. The RTC reconstructs the wavefront and calculates the voltages sent to the DM to compensate the distorted wavefront. Meanwhile, the scientific camera records the corrected images and sends them for post-processing in order to get even higher resolution.
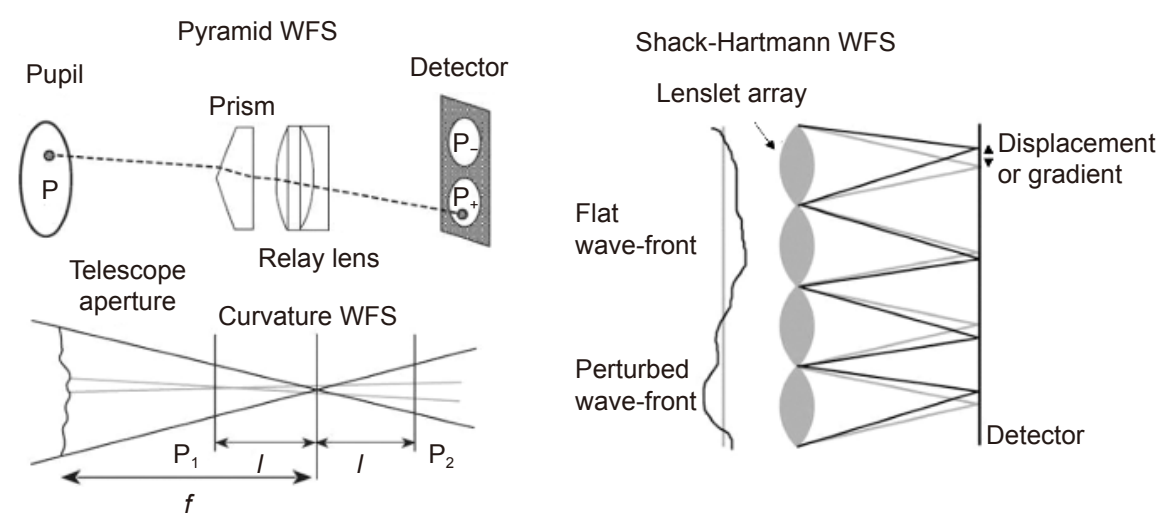

Fig. 2 | Principles of three kinds of WFS. Figure reproduced with permisson from ref. ${ }^{32}$, Annual Reviews Inc.

millisecond time-scale speed required by the AOS. On the other hand, these WFSs have complex optical setup and require high accuracy alignment. Besides, some demands such as continuous wavefront or uniform amplitude must be met for these gradient or curvature-based methods. These requirements can be broken in some extreme observation circumstances when the target is at low-elevation or the turbulence is too strong and then the performance would deteriorate quickly.

\section{Traditional wavefront reconstruction and control}

Wavefront reconstruction and control builds the relationship between DM commands and the WFS measurements. The wavefront here can be represented as a 2-dimensional phase map as well as modal coefficients of some bases such as the Zernike modes ${ }^{36}$, Karhunen-Loève modes ${ }^{37}$ or the so-called Nodal modes ${ }^{38}$. The wavefront reconstruction spatially filters the WFS measurements and recovers the closed-loop wavefront error while the wavefront control temporally filters the wavefront error and drives the DM. The most popular control in $\mathrm{AO}$ is the class of proportional-integral-derivative (PID) algorithms including the integrator or the proportional-integral (PI) control et $\mathrm{a}^{39}$. However, innumerable researches have shown that PID is not the optimal choice for AOSs, because its poor performance for the correction of dynamic turbulence and narrowbandwidth disturbances. Linear quadratic Gaussian (LQG) control based on Kalman filter is an appealing control strategy ${ }^{40-44}$. It can obtain an optimal correction in terms of residual phase variance by performing optimal prediction of dynamic disturbance. The key of this method is to quickly and consecutively build the accurate dynamical model to track the time-varying dynamic 
disturbance ${ }^{45,46}$. LQG is a kind of linear prediction algorithm in which any deviation from the estimated model would degrade the performance.

\section{Traditional post-facto image reconstruction}

The AOS can only partially correct the distortions in real time due to the unavoidable inherent errors of the system. The residual errors which are composed by the measurement error, fitting error, time-delay induced error, and anisoplanatism error et al. decrease the quality of the imaging. Thus, post-facto processing techniques are required to reach diffraction limit of the system.

The image of the astronomical object $i(x)$ can be expressed mathematically as the convolution of the object intensity $o(x)$ and the point spread function (PSF) of the whole atmosphere + telescope + instruments system $p(x)$ :

$$
i(x)=o(x) * p(x) .
$$

In the Fourier domain the equation is transformed to

$$
I(v)=O(v) \cdot P(v),
$$

where the upper characters are defined as the Fourier transform of the lower cases, and $v$ is the index at the frequency domain. To deconvolve the object intensity $o(x)$ from the observed intensity $i(x)$, the PSF of the system is needed. The precise PSF is rarely known in actual system, except that the residual wavefront is measured or there is an unresolved star within the isoplanatic angle. Thus, the blind deconvolution (BD) ${ }^{47-49}$ is proposed using only the prior assumption of the system to simultaneously obtain the object and the PSF. The BD is illdefined due to the deficiency of the information about the actual system and thus the accuracy of the reconstruction depends on the rationality of the assumed priors.

Another comparable method is the phase diversity (PD) method ${ }^{50-52}$ which can be considered as a special case of the BD. With an additional information on the phase (usually using the defocus of one image), the method can detect the residual wavefront simultaneously and can be used for wavefront sensing in the AOS.

Adding more information of the system can lessen the ambiguity of the BD method and get more reliable result. The multi-frame BD (MFBD $)^{53-55}$ and the multi-object MFBD (MOMFBD) ${ }^{56}$ are then introduced. By assuming the observed object is not changed during the time used to capture the multiple image frames, the solutions of the method are more robust than the BD. The MFBD is fur- ther extended to include the multiple objects case. The MOMFBD consists of a maximum-likelihood solution to the deconvolution problem which is widely used in the case of simultaneously reconstructing the broadband and narrow-band channel images at the solar observations.

The statistical information of the turbulence can also be applied in the reconstruction of the astronomical object. The speckle imaging method uses the short exposure images to recover the Fourier amplitude and the Fourier phase of the object separately. In the process, the Fried parameter of the turbulence is estimated and the speckle transfer function of the system which is the power spectrum of the PSF is deduced using the correction abilities of the AOS and the Fried parameter of the turbulence ${ }^{57,58}$. The Labeyrie's method is used to deconvolve the Fourier amplitude of the object ${ }^{59}$. The triplecorrelation bispectra method ${ }^{60-62}$ and the KnoxThompson cross-spectrum method ${ }^{63}$ reconstruct the Fourier phase of the object. The reconstructed objects are obtained by inverse transforming the Fourier amplitude and the Fourier phases.

The high photometric accuracy of the MOMFBD method and speckle imaging method are confirmed by their wide applications on the reconstructions of the astronomical objects at nearly all the ground-based observation sites ${ }^{64-67}$. But the drawbacks of those method are the necessary computing effort and memory for the masses of iterations within the process. To get a real time reconstruction, those methods usually require the use of a dedicated computer cluster or similar installations ${ }^{68,69}$. However, the processing time can be significantly decreased using the intelligent image reconstruction methods described in Subsection Intelligent adaptive optics.

\section{Intelligent adaptive optics}

AOS by its name has already shown some degree of intelligence. In this review, we distinguish the intelligence from non-intelligence by the application of machine learning techniques which can learn rules from data. IAO is a technique that reshapes the wavefront aberration measurement, control and post-processing through machine learning to improve the performance of $\mathrm{AO}$ or simplify the system complexity. Recent advancements in IAO are summarized in this section.

\section{Intelligent wavefront sensing}

SHWFS is the most widely used WFS in AOS due to its 
simple structure, alignment and less computation. However, the reconstructed result is sensitive to noise. Besides of improving the measurement accuracy of SHWFS by optical modulation ${ }^{70}$, advanced algorithms can also be used. Two kinds of machine learning-based methods are proposed to improve the SHWFS's performance. One is to improve the gradient-based method such as building the relationship between aberrations and gradients with nonlinear fitting tools such as ANN instead of simple matrix multiplication, or improving the spot centroid accuracy by doing the spot classification with ANN before centroid calculation. The other is to extract the aberration from the SHWFS image directly with deep learning instead of calculating the gradients. In some applications, traditional special WFSs are not allowed and the imaging setup can be used as the PD WFS or single-image phase retrieval WFS. In these cases, deep learning can be used to solve the nonlinear phase retrieval problem without many iterations required by traditional Gauss-Seidel or stochastic parallel gradient descent (SPGD) methods etc. Besides of improving accuracy and speed of traditional narrow field-of-view WFS, deep learning can also be used to improve the performance of tomography WFS.

\section{Shack-Hartmann wavefront sensor}

Generally, wavefront is calculated from sub-aperture spot displacements with LSF or SVD in SHWFS. Guo et al proposed to use ANN to reconstruct the wavefront from noisy spot displacements after comparing the performances of LSF, SVD and ANN. The lens array of the SHWFS was assumed to consist of $8 \times 8$ sublenses. Different structures of neural network were investigated to find the optimal network architecture. For training on the noisy patterns, the best network was a three-layer feed forward back-propagation network with 90 neurons in the hidden layer. The principle of training and simulation result are shown in Fig. 3. After training on the noisy patterns, the residual error of ANN is much smaller than the other two methods ${ }^{71}$. Besides of ANN, Swanson et al proposed to use a U-Net architecture to learn a mapping from SHWFS slopes to the true wavefront in 2018, but this method was not compared with others $^{72}$.

To overcome the strong environment light and noise pollutions, Li et al. proposed another method based on ANN, namely SHWFS-Neural Network (SHNN), as described in Fig. 4. In this method, SHNNs firstly find out the spot center, and then calculate the centroid, which transform spot detection problem into a classification problem. As shown in Table 1, when the signal-to-noise ratios (SNRs) are interfered by the environment light and ramp in the subaperture images, SHNNs show stronger robustness compared with other methods, which means this method can be used in AOSs under extreme conditions ${ }^{73}$.

To avoid any information loss during the centroid calculating procedure in off-axis WFSs, Suárez Gómez et al.
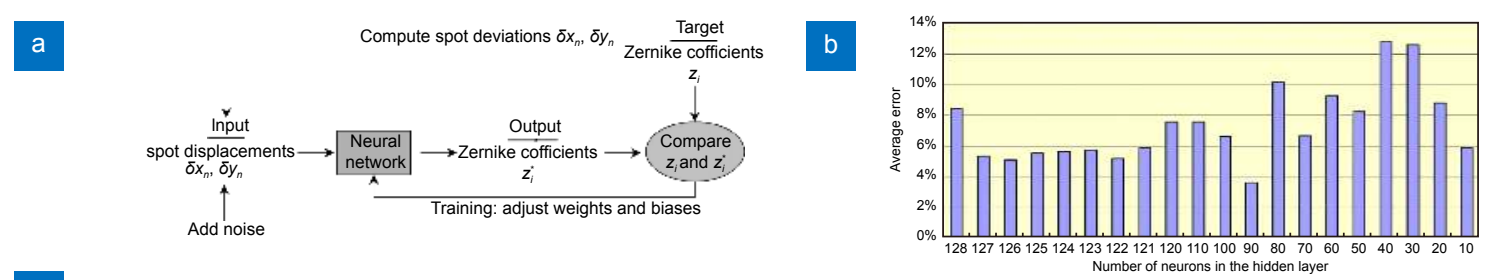

c
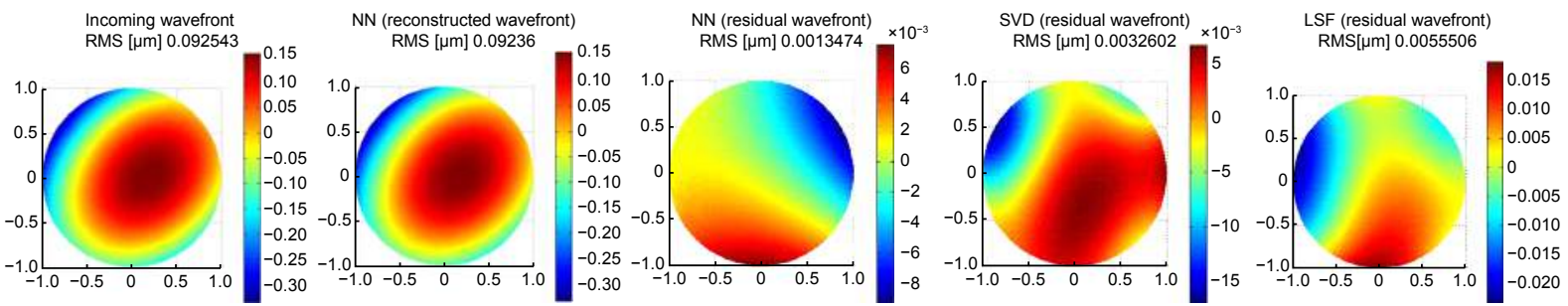

Fig. 3 | (a) The training algorithm of the ANN where displacements of the spots are taken as the inputs while the Zernike coefficients as outputs. To make the network more insensitive to the noise in the spot patterns, the training is also performed on noisy patterns. The noise added to the spot displacements follows Gaussian distribution. (b) The average reconstructed errors of ANNs with different number of neurons in the hidden layer shows that hidden layer with 90 neurons performs best. (c) Comparison of residual errors of LSF, SVD and ANN algorithms. Figure reproduced from ref. ${ }^{71}$, Optical Society of America. 


\section{a}

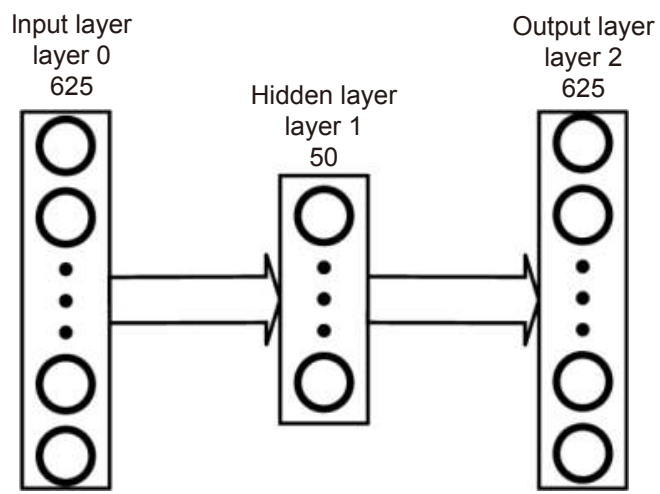

b
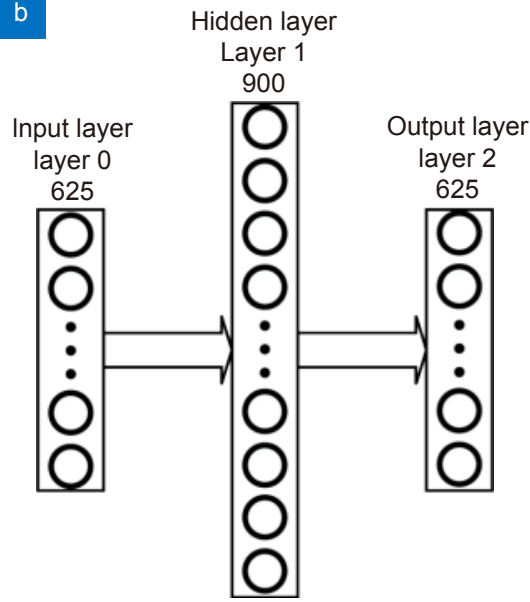

Fig. 4 | (a) The classification network similar to CoG method for spot detection with 50 hidden layer neurons named as SHNN-50. (b) The classification network with 900 hidden layer neurons named as SHNN-900. The input is the flattened subaperture image $(25 \times 25)$ and the output is a kind of classification in 625 classes, the same as the number of pixels indicating the potential center of the spot. Figure reproduced from ref. ${ }^{73}$, Optical Society of America.

Table 1 | The false rate of different methods in low SNR situations, where CoG means Center of Gravity method and TmCoG means a modified CoG method using $\mathrm{m} \%$ of the maximum intensity of spot as threshold. Table reproduced from ref. ${ }^{73}$, Optical Society of America.

\begin{tabular}{c|c|c|c|c|c}
\hline \multirow{2}{*}{ SNR $_{P}$} & CoG & Windowing & TmCoG & SHNN-50 & SHNN-900 \\
\cline { 2 - 6 } & 98 & 83 & 94 & 73 & 55 \\
\hline 1 & 97 & 43 & 61 & 28 & 7 \\
2 & 97 & 28 & 26 & 6 & 0 \\
3 & 98 & 8 & 6 & 1 & 0 \\
4 & 97 & 2 & 1 & 1 & 0 \\
\hline
\end{tabular}

presented a method using convolutional neural network $(\mathrm{CNN})$ as a reconstruction alternative in $\mathrm{MOAO}^{74}$. As displayed in Fig. 5(a), this method relies on the use of the full image as input, instead of only the centroids. Simulations have shown the advantages of CNN compared with the multi-layer perceptron (MLP) as shown in Fig. 5(b). The promising results of CNN open possibilities in further work in the topic, such as improving the topology of the network, setting more solid testing with sets of multilayer turbulence profiles and using optical measurements for the comparison of errors.

Although Suárez Gómez et al use the whole off-axis SHWFS images as input, they still calculate the slopes of the on-axis target and use these slopes to reconstruct the wavefront. However, calculating only the centroids or slopes dose not overcome the limitations of the wavefront reconstruction using slope features. To further improve the performance of SHWFS, DuBose et al. ${ }^{75}$ extended the work of Swanson et al. ${ }^{72}$ and developed the Intensity/Slopes, or ISNet, a deep convolution network utilizing both the standard SHWFS slopes and the total intensity of each subaperture to reconstruct aberration phase. The architecture of the ISNet is shown in Fig. 6(a). The main difference between ISNet and the work of Swanson et al. is the use of intensity and dense blocks. Four reconstruction algorithms (ISNet, ISNet without intensity, Swanson et al.'s work and the Southwell Least Squares) are compared in ref. ${ }^{75}$. The results are shown in Fig. 6(b). The ISNet offers superior reconstruction performance.

In biological applications, deep learning has been used to detect high-order aberration directly from SHWFS images without image segmentation or centroid positioning. $\mathrm{Hu}$ et al. proposed a method named learningbased Shack-Hartmann wavefront sensor (LSHWS) which could predict up to $120^{\text {th }}$ Zernike modes with a SHWFS image as input ${ }^{76}$. The architecture of the LSHWS is displayed in Fig. 7. The compensation results of LSHWS and Traditional Shack-Hartmann wavefront sensor (TSHWS) are given in Table 2. The correlation coefficient of LSHWS corrected patterns is $\sim 5.13 \%$ higher than that of TSHWS. In addition, $\mathrm{Hu}$ et al. ${ }^{77}$ also 
a
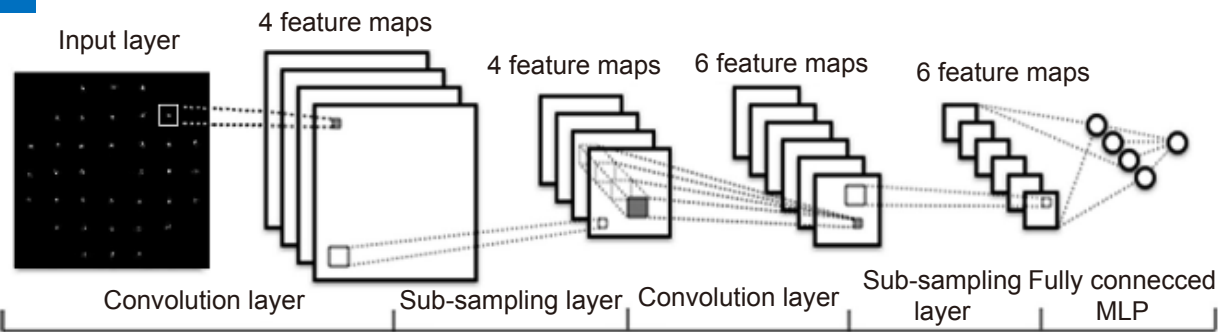

b

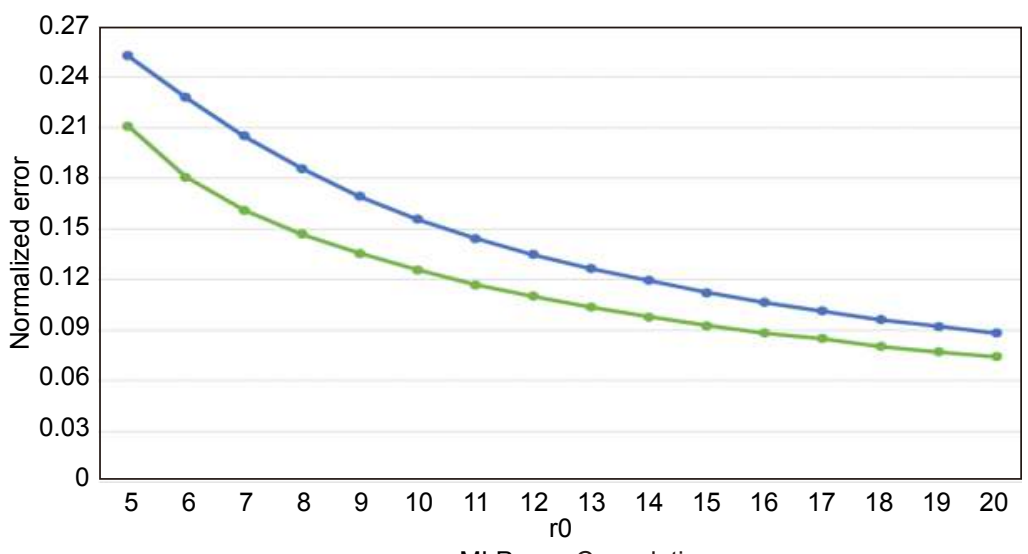

Fig. 5 | (a) The architecture of the CNN for SHWFS. The input is a SHWFS image and the output is a vector, which indicates centroids. (b) The performance of CNN compared with MLP where the error is calculated as the average of the absolute value of the difference between all the output network centroids and the simulated true centroids. Figure reproduced from: (a, b) ref. ${ }^{74}$, International Conference on Hybrid Artificial Intelligence Systems.

\section{a}

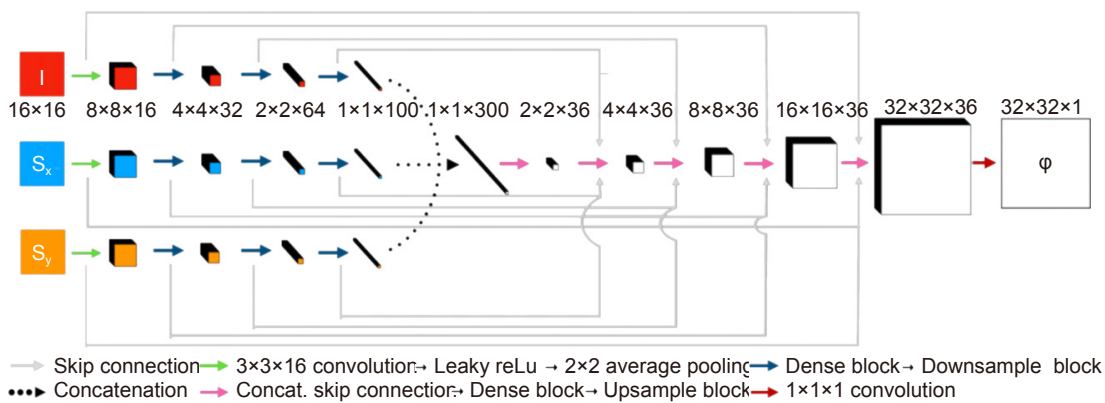

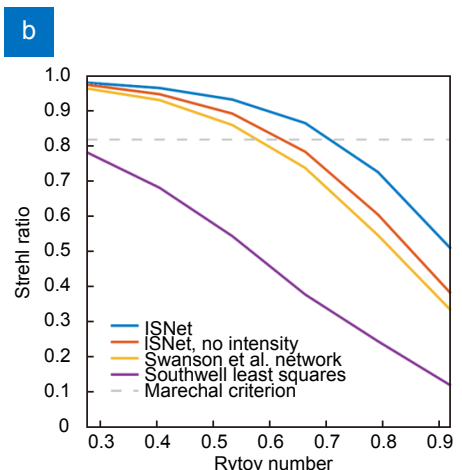

Fig. 6 | (a) Architecture of ISNet. This network takes three $16 \times 16$ inputs ( $x$-slopes, $y$-slopes and intensities) and outputs a $32 \times 32$ unwrapped wavefront. (b) Plot of average Strehl ratio vs. Rytov number for different reconstruction algorithms. For comparison, the Strehl ratio of a Marèchal criterion-limited beam is shown in (b), which is 0.82 . Figure reproduced from: $(a, b)$ ref. ${ }^{75}$, Optical Society of America.

proposed another deep learning assisted SHWFS named $\mathrm{SH}-\mathrm{Net}$ which could directly predict the wavefront distribution without slope measurements or Zernike modes calculation. The architecture of SH-Net is shown in Fig. 8(a) and 8(b). The detection speeds of different methods are given in Table 3. From Fig. 8(c) and Table 3, we can see that $\mathrm{SH}-\mathrm{Net}$ has the highest accuracy among the five methods. Although the detection speed of the SH-Net is slower than approach of Swanson et al. and zonal, the accuracy is much higher.

\section{Phase diversity wavefront sensor}

Compared with SHWFS, image-based wavefront sensing is a method without additional optical components but using parameterized physical model and nonlinear optimization. PD WFS is such a type of image-based WFS. As early as 1994, Kendrick et al. used general regression neural network to calculate the wavefront errors from focused and defocused images ${ }^{78}$. Recently, with the development of artificial intelligence, many new methods based on CNN for wavefront detection have been 


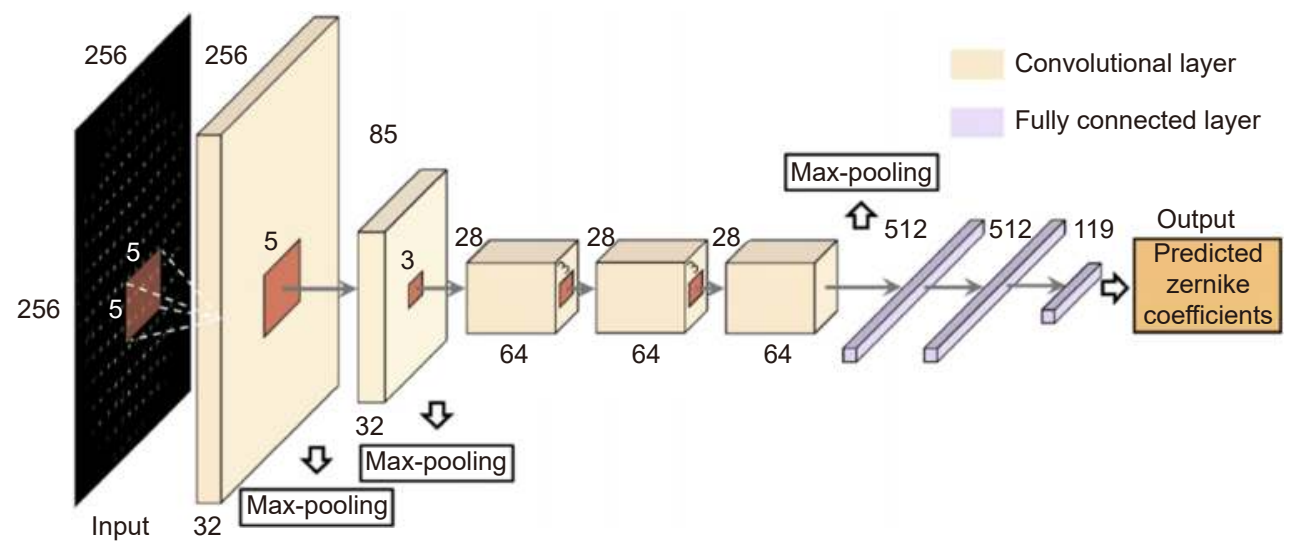

Fig. 7 | Architecture of LSHWS. The network contains five convolutional layers and three full connected layers. The input is a SHWFS image of size $256 \times 256$. The output is a vector of size 119 , which represents Zernike coefficients. Figure reproduced from ref. ${ }^{76}$, Optical Society of America.

Table 2 | The correlation coefficient of SHWFS patterns and the Strehl ratio of PSFs. Table reproduced from ref. ${ }^{76}$, Optical Society of America.

\begin{tabular}{c|c|c|c|c}
\hline \multirow{2}{*}{ Correction } & \multicolumn{2}{|c}{ Correlation coefficient } & \multicolumn{2}{c}{ Strehl ratio } \\
\cline { 2 - 5 } & Mean & Std.Dev. & Mean & Std.Dev. \\
\hline Distorted & 0.5178 & 0.0245 & 0.1201 & 0.1702 \\
\hline TSHWS & 0.8863 & 0.0093 & 0.7775 & 0.0707 \\
\hline LSHWS & 0.9318 & 0.0039 & 0.9898 & 0.0238 \\
\hline
\end{tabular}
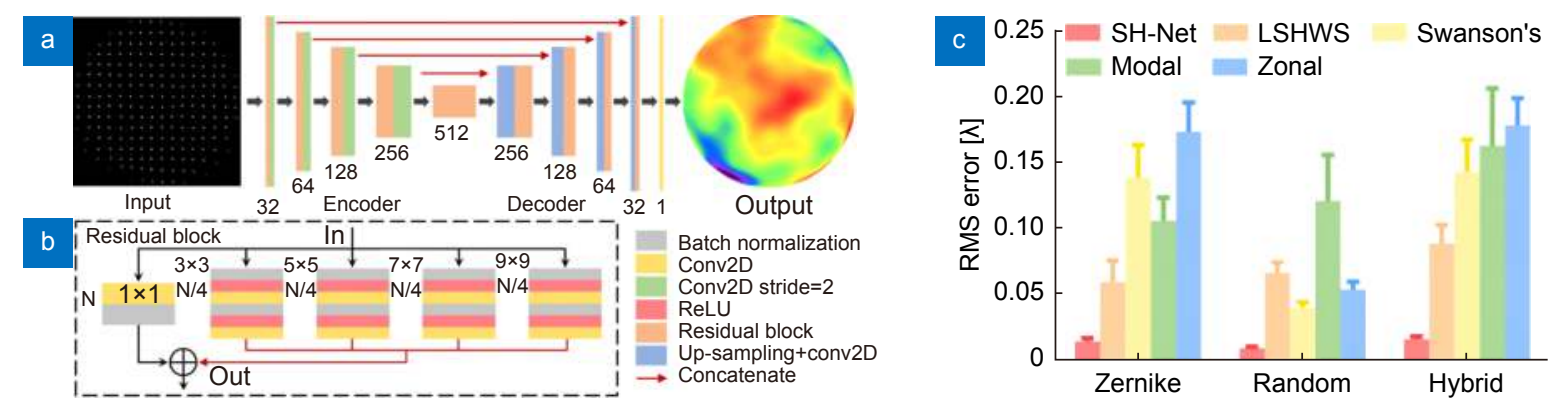

Fig. 8 | (a) Architecture of SH-Net. The input is a SHWFS image of size $256 \times 256$, and the output is a phase map with the same size as the input. (b) the Residual block. ' $\mathrm{N}$ ' and ' $\mathrm{N} / 4$ ' indicate the number of channels. (c) Statistical results of RMS wavefront error of five methods in wavefront detection. Figure reproduced from: (a-c) ref. ${ }^{77}$, Optical Society of America.

proposed. J R. et al. used machine learning to determine a good initial estimate of the wavefront. Although it can improve the effectiveness of the traditional gradient descent algorithm, iterative operations are still required ${ }^{79}$. Guo et al. used an improved CNN to successfully establish the nonlinear mapping between the focal/defocused PSFs and the corresponding phase maps ${ }^{80}$. As shown in Fig. 9, their deconvolution visual geometry group network (De-VGG) adds three deconvolution layers on the basis of the well-known visual geometry group (VGG) network. Compared with SPGD algorithm, De-VGG has a great advantage in running time. The inference time of SPGD and De-VGG is shown in Table 4.

Ma et al. proposed a similar PD wavefront sensing technique to Guo et al.'s ${ }^{80}$ meanwhile, as shown in Fig. $10^{81}$. There are two CCDs for detecting intensity images.
The AlexNet is used to extract the features from the focal and defocused intensity images and obtain the corresponding Zernike coefficients. Figure 10(c) shows the Strehl ratio of CNN compensation under different SNR conditions. When the SNR reaches 50 or $35 \mathrm{~dB}$, the compensation results are almost coincided and the system is robust. When the SNR reaches $20 \mathrm{~dB}$, the robustness of the system reduces and the results fluctuate greatly. As no real data or experiments were used to investigate the accuracy, it is not clear about the robustness of this method.

$\mathrm{Wu}$ et al. proposed a novel real-time non-iterative phase-diversity wavefront sensing based $\mathrm{CNN}$ that achieves sub-millisecond phase retrieval ${ }^{82}$. Figure 11 shows two parts of the experiments. This method improves the real-time performance by using NVIDIA 
Table 3 | The detection speed of five methods. Table reproduced from ref. ${ }^{77}$, Optical Society of America.

\begin{tabular}{c|c|c|c|c|c}
\hline & SH-Net & LSHWS & Swanson's & Modal & Zonal \\
\hline Detection speed & $40.2 \mathrm{~ms}$ & $0.1225 \mathrm{~s}$ & $22.6 \mathrm{~ms}$ & $0.1294 \mathrm{~s}$ & $26.3 \mathrm{~ms}$ \\
\hline
\end{tabular}

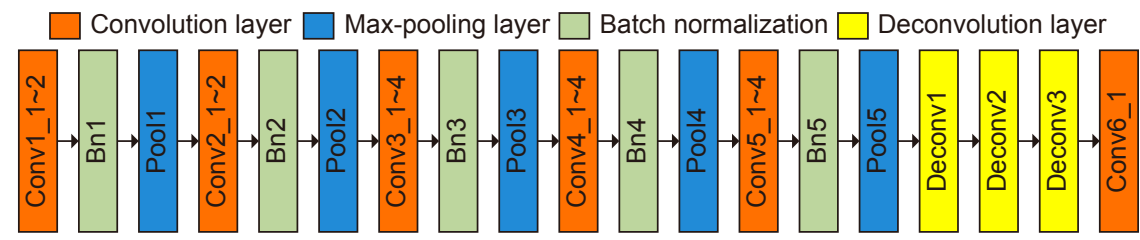

Fig. 9 | Architecture of the deconvolution VGG network (De-VGG). The De-VGG includes convolution layers, batch normalization layer filters, activation function ReLU, and deconvolution layers. The fully connected layers are removed, as the high output order slows down the calculation speed, but the deconvolution layer will not. Figure reproduced from ref. ${ }^{80}$, under a Creative Commons Attribution License 4.0.

Table 4 | The inference time of De-VGG compared with SPGD. Table reproduced from ref. ${ }^{80}$, MDPI.

\begin{tabular}{c|cc}
\hline & SPGD $(\mathrm{ms})$ & De-VGG (ms) \\
\hline$D / r_{0}=6(\mathrm{RMS}=0.0703 \lambda)$ & 304 & 11 \\
$D / r_{0}=10(\mathrm{RMS}=0.0703 \lambda)$ & 448 & 11 \\
\hline
\end{tabular}

a

Two intensity images data sets

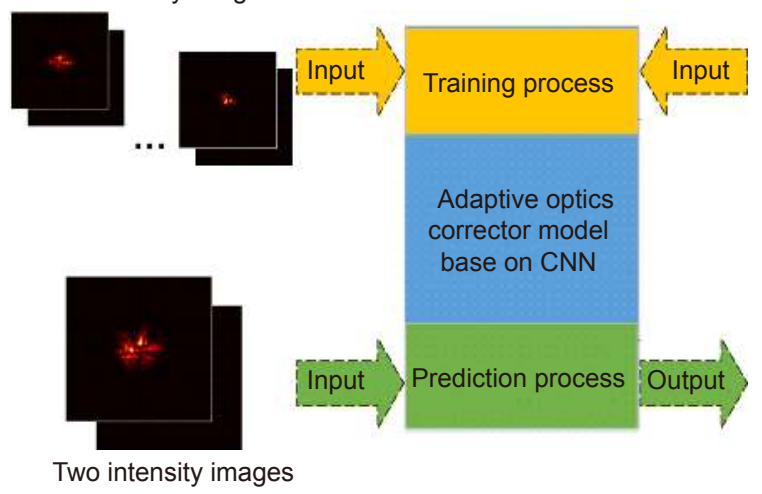

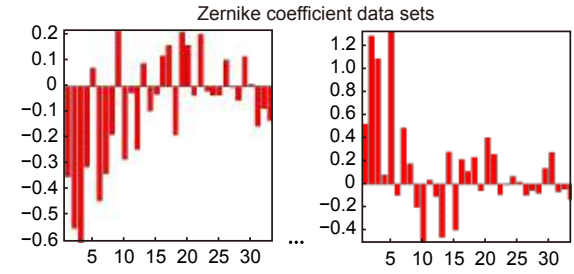

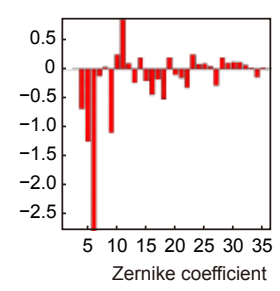

b

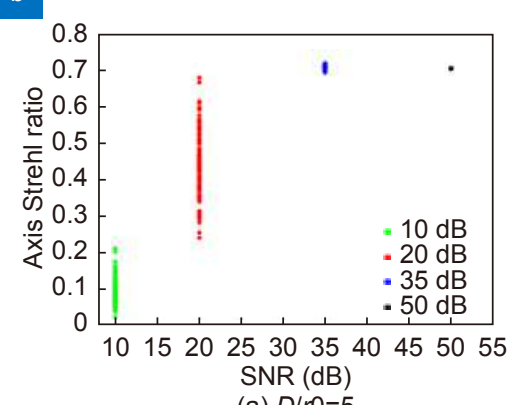

(a) $D / 10=5$

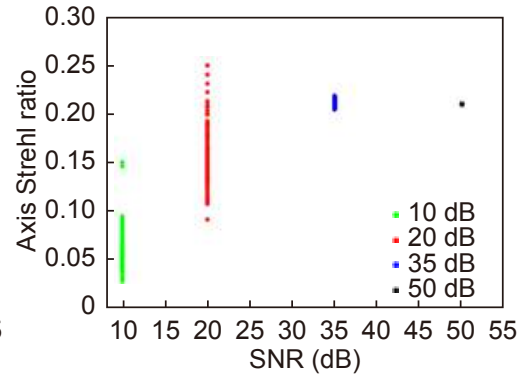

(b) $D / r 0=10$

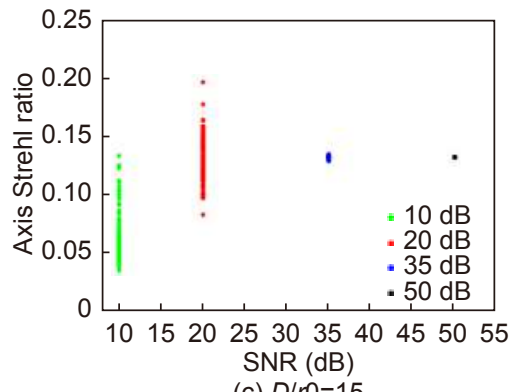

(c) $D / 10=15$

Fig. 10 | (a) The data flow of the training and inference processes. (b) Strehl ratio of CNN compensation under different SNR conditions. Figure reproduced with permission from: $(a, b)$ ref. ${ }^{81}$, Elsevier.

TensorRT (an SDK for high-performance deep learning inference) and reduces the aberration measurement error by fusing the focal and defocused intensity images. However, there is some accuracy loss after inference acceleration (Table 5). It is necessary to further study hardware optimization principles of TensorRT.

\section{Phase retrieval from single image}

To further simplify the optical setup, Nishizaki et al. proposed a variety of image-based wavefront sensing architectures named deep learning WFS (DLWFS) that could directly estimate aberration from single intensity image using Xception ${ }^{83}$. As shown in Fig. 12, this method 

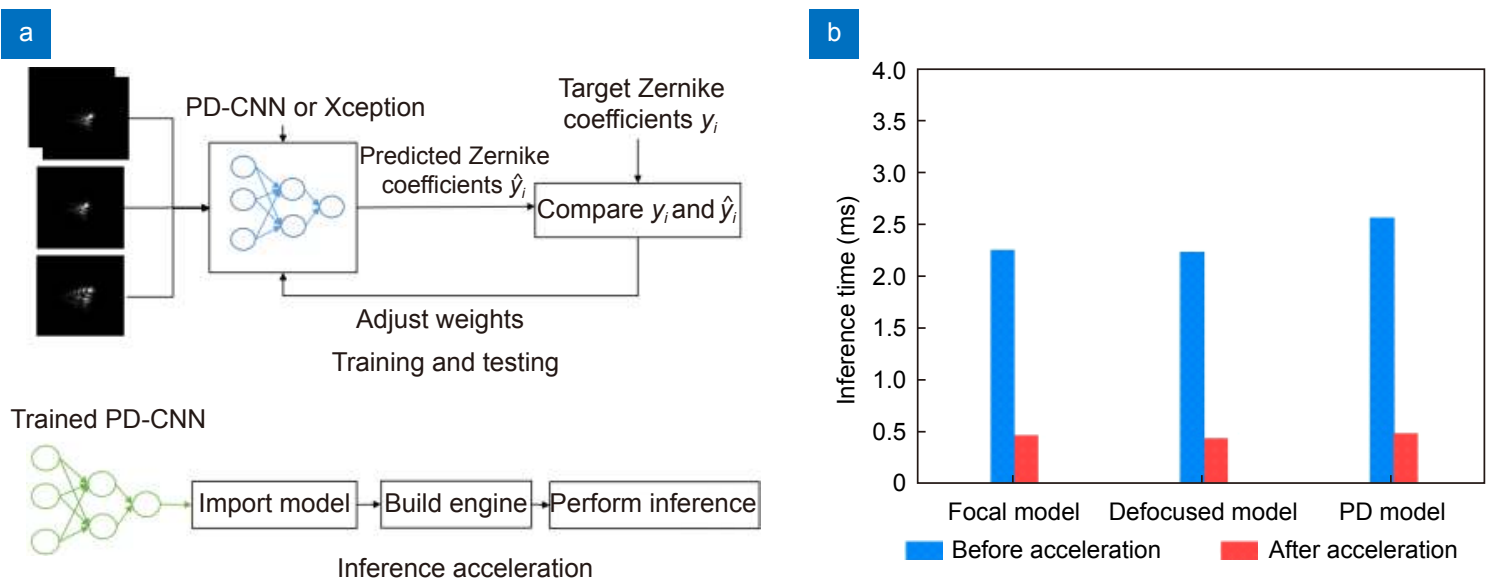

Fig. 11 | (a) The experiments of training and testing processes and inference acceleration. During training and testing processes, two networks were used, phase-diversity CNN (PD-CNN) and Xception. PD-CNN includes three convolution layers and two full connection layers. For each network, three sets of comparative experiments are set as following, inputting the focal and defocused intensity images separately, and inputting the focal and defocused intensity images at the same time. During the inference acceleration process, the trained model is optimized by TensorRT. (b) The inference time before and after acceleration with NVIDIA GTX 1080Ti. Figure reproduced from: (a, b) ref. ${ }^{82}$, under a Creative Commons Attribution License 4.0.

is suitable for both point and extended sources and the types of preconditioners include overexposure, defocus and scatter. The results in Table 6 show that all of the mentioned preconditioners can vastly improve the estimation accuracy when performing in-focus imagebased estimation and among them overexposure is the optimal. For further study, it is important to compare the DLWFS with conventional WFSs to validate its usefulness as a practical replacement.

\section{Tomography wavefront sensing}

Traditional single conjugate AOS can only work when the scientific target is near a bright guide star or the scientific target itself is bright enough. These requirements limit the number of stars on the sky that can be observed with high resolution. The majority of modern AOSs use tomographic reconstruction techniques to overcome this problem. The mostly investigated configurations are laser tomography AO, MCAO and MOAO.

MOAO is a large-field-of-view AO technique which

Table 5 | Summary of accuracies (Root Mean Square Error (RMSE): $\lambda$ ) of Zernike coefficients estimated by PD-CNN. The focal model and defocused model mean the input of PD-CNN is a single focal intensity image or defocused intensity image. The PD model means that the input of PD-CNN includes both focal and defocused image. Table reproduced from: $(a, b)$ ref. ${ }^{82}$, MDPI.

\begin{tabular}{c|c|c|c|}
\hline & Focal model & Defocused model & PD model \\
\hline Before acceleration & $0.1004 \pm 0.0469$ & $0.0823 \pm 0.0492$ & $0.0529 \pm 0.0286$ \\
\hline After acceleration & $0.1142 \pm 0.1119$ & $0.0974 \pm 0.1137$ & $0.0696 \pm 0.1092$ \\
\hline
\end{tabular}

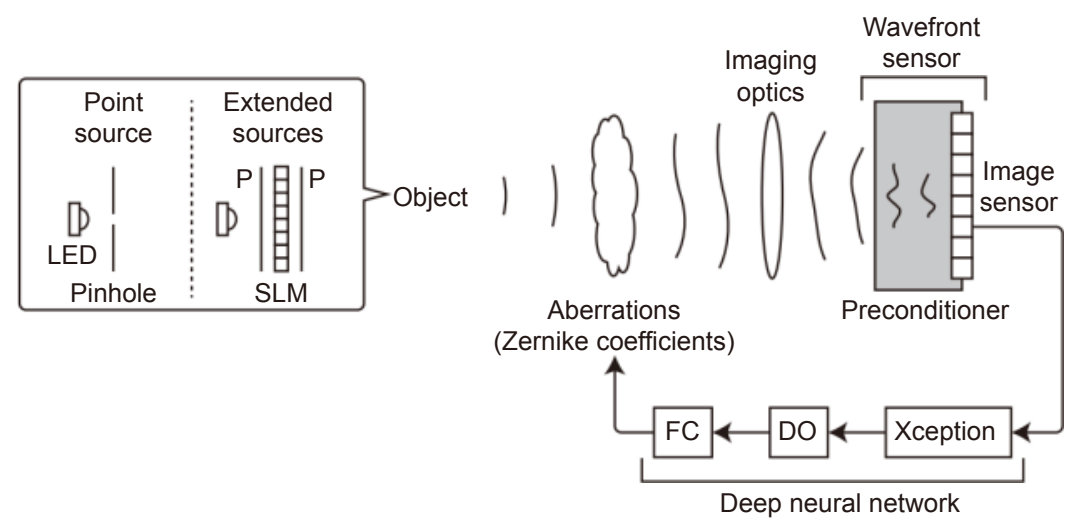

Fig. 12 | Schematic and experimental diagram of the deep learning wavefront sensor. LED: light emitting diode. P: Polarizer. SLM: Spatial light modulator. DO: Dropout layer. FC: full connection layer. Figure reproduced from ref. ${ }^{83}$, Optical Society of America. 
Table 6 | Summary of accuracies (RMSE: $\lambda$ ) of Zernike coefficients estimated by Xception. Table reproduced from ref. ${ }^{83}$, Optical Society of America.

\begin{tabular}{c|c|c|c|c}
\hline & In-focal & Overexposure & Defocus & Scatter \\
\hline Point source & $0.142 \pm 0.032$ & $0.036 \pm 0.013$ & $0.040 \pm 0.016$ & $0.057 \pm 0.018$ \\
\hline Extended source & $0.288 \pm 0.024$ & $0.214 \pm 0.051$ & $0.099 \pm 0.064$ & $0.195 \pm 0.064$ \\
\hline
\end{tabular}

simultaneously measures the open loop wavefront of several distributed guide stars and estimates the phase aberration of each target. Osborn et al. proposed a MLP named CARMEN to combine the information from the off-axis WFS slopes and output the on-axis wavefront slopes from the target to the telescope ${ }^{84}$. The MLP diagram and experimental result of CARMEN are shown in Fig. 13. As demonstrated by the results, the L\&A tomographic reconstruction outperforms the CARMEN by approximately $5 \%$ in Strehl ratio. However, CARMEN is considered to be more robust when the altitude of high layer turbulence changes because in L\&A, the on-line measurements from all of the WFSs must be combined and theoretical functions are used to recover the turbulence profile. Moreover, the experiment also proved that off-line training using simulation data can be used for realistic situations.

\section{Intelligent wavefront prediction}

Due to the exposure and readout time required by the WFS, the reconstruction and control calculation time cost by the RTC and the response time of the DM, AOS usually suffers about $2 \sim 3$ frames time delay. Traditional control algorithms don't consider the wavefront distortion change from measurement to correction so there is a so-called time-delay error. One of the effective methods to reduce this time-delay error is to predict the future wavefront with several previous frames.

As firstly demonstrated by Jorgenson and Aitken that astronomical wavefront can be predicted ${ }^{85}$, numerous efforts have been done to improve the prediction accuracy. At first, linear predictors such as the linear minimum mean square error (LMMSE) algorithm based on the statistical knowledge of the atmosphere, noise etc. were investigated, with the advantages of simple architecture and less computations ${ }^{86}$. However, the accurate a priori knowledge about the atmospheric turbulence and noise cannot be obtained easily in real systems, especially for non-stationary turbulences. For instance, as shown in Fig. 14, the varying wind speed has a significant impact on the performance of prediction, preventing it from reaching optimal performance ${ }^{87}$.

Two classifications of algorithms are expected to solve this problem, one is to estimate the statistical properties in quasi real-time ${ }^{88}$ and the other is to train the predictor with big data to adapt the variation of the turbulence. The machine learning based predictor belongs to the second one. As early as 1997, Montera et al compared the LMMSE estimator with neural network estimator and drew the conclusion that the neural network could outperform the LMMSE when the seeing varies over a range of conditions ${ }^{31}$. Early studies about prediction using neural networks were usually based on the feed-forward MLP network. With the development of deep learning,
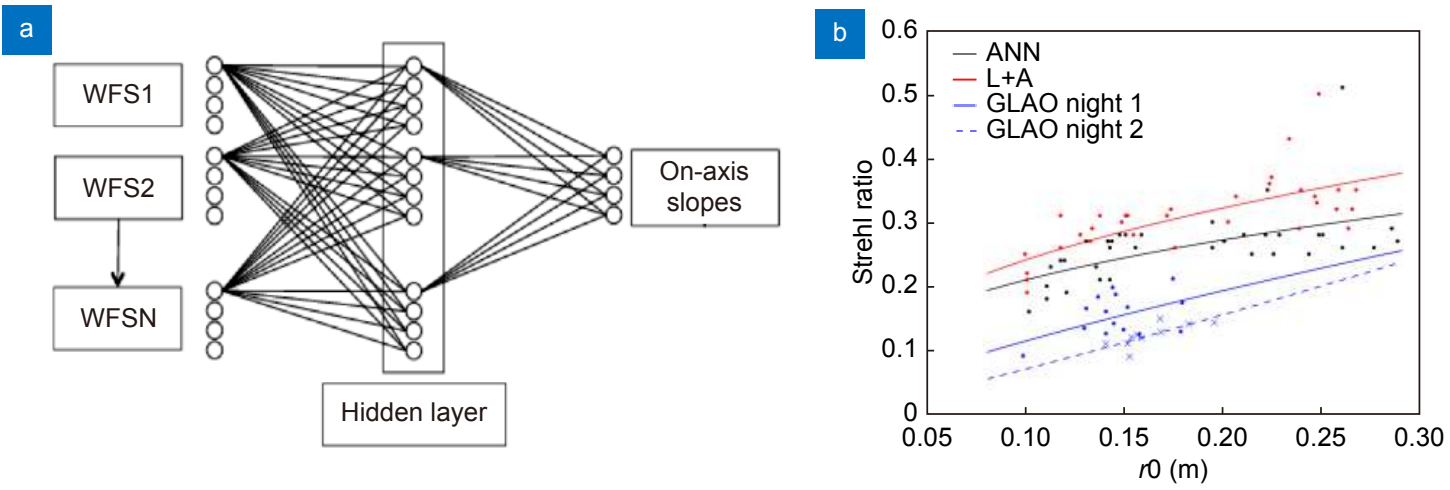

Fig. 13 | (a) Network diagram for CARMEN. where the inputs are the slopes of off-axis WFSs and the outputs are the on-axis slopes for the target direction. One hidden layer with the same number of neurons as the inputs is used to link the inputs and outputs and the sigmoid activation function is used. (b) On-sky Strehl ratio (in H-band) comparison with different methods. This on-sky experiment was carried out on the 4.2m William-Herschel Telescope. The Strehl ratios achieved by the ANN (CARMEN), the Learn and Apply (L\&A) method and two GLAO night performances are compared. Figure reproduced from $(a, b)$ ref. ${ }^{84}$, Oxford University Press. 
recurrent neural networks having dynamic feedback connections and sharing parameters across all time steps are better for sequence data processing tasks like wavefront prediction. Long short term memory (LSTM) has been extensively studied to predict the turbulence induced wavefront ${ }^{72,89,90}$. As shown in the Fig. 15, Liu et al. showed that LSTM had the ability to learn information such as wind velocity vectors from the data and could use this information for prediction in open-loop AOS ${ }^{91}$. Liu emphasized that the selection of training regime was very important for the performance of ANN's prediction. This means that the training data and methods play a great role in machine learning-based prediction. To fight against overfitting and improve the generalization capability, Sun et al. proposed a Bayesian regularization back propagation algorithm to make the tradeoff between the fitting error and model complexity in the objective function ${ }^{92}$.

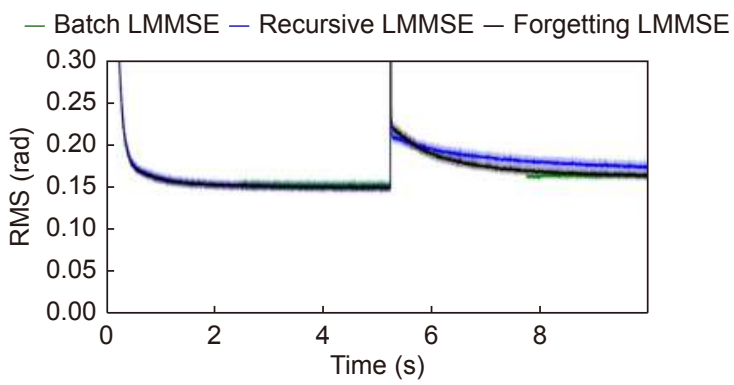

Fig. 14 | Wind jumps of $7 \mathrm{~m} / \mathrm{s}$ showing the convergence of the recursive LMMSE (blue) and the forgetting LMMSE (black), as well as the resetting of the batch LMMSE (green) for a regressor with a 3-by-3 spatial grid and five previous measurements for each phase point. Figure reproduced from ref. ${ }^{87}$, Optical Society of America.

It can be anticipated that the prediction of atmospheric distorted wavefront will be a research focus in the near future especially for high contrast $\mathrm{AO}^{93}$ and low-flux $\mathrm{AO}^{94}$. Deep learning techniques have superior advantages over linear predictors when dealing with real nonstationary turbulence. However, several problems have to be analyzed and tested before applying in real AOS. One is the impact of error introduced by additional noise and nonlinear response of the system on prediction accuracy when pseudo open-loop slopes have to be used in real closed-loop AOS. Another is the trade-off between prediction accuracy and calculation amount for real-time operation of the RTC. Besides, the most important task for machine learning-based prediction algorithm development seems to collect enough high-quality training data for better generality of working with real time-varying turbulence. Whether simulated data can be used to train predictors used on-sky is worth studying.
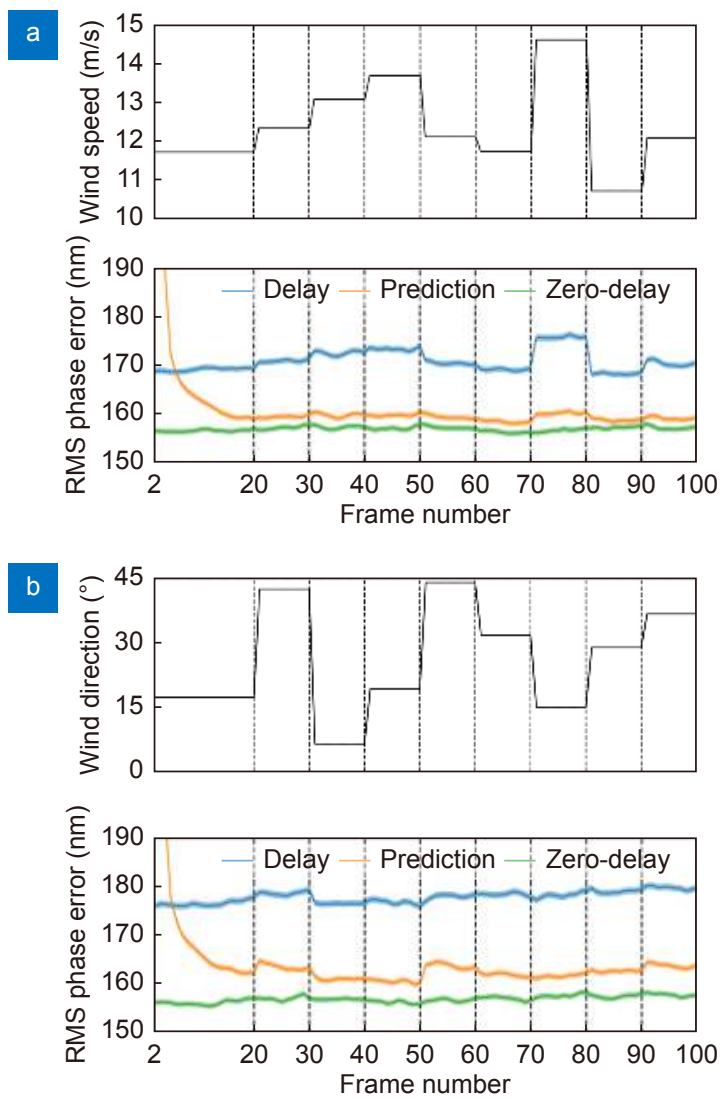

Fig. 15 | (a) Robustness of the predictor against wind speed fluctuations between 10 and $15 \mathrm{~m} / \mathrm{s}$ every 10 frames. (b) Robustness of the predictor against wind direction fluctuations between 0 and 45 degrees every 10 frames. Figure reproduced from: $(a, b)$ ref. ${ }^{91}, \mathrm{Ox}-$ ford University Press.

\section{Intelligent post-processing}

One attractive advantage of the machine learning technique is the rapid processing rate once the model is trained. In the reconstruction of the astronomical objects, machine learning can dramatically decrease the computing time compared with traditional methods. With suitable computer hardware, it is anticipated that on-site real-time reconstruction of astronomical images will be possible in the near future.

Based on the theoretical basis of the MFBD, two different deep learning architectures were proposed by Ramos et al. in $2018^{95}$, which are shown in Fig. 16. The first one (Fig. 16(a)) fixes the number of inputting frames and uses them as channels in a standard CNN which is an end-to-end approach based on an encoderdecoder network. The output of the $\mathrm{CNN}$ is the corrected 
frame, taking into account all the spatial information encoded in the degraded frames. To process a larger number of frames, one applies the deep neural network (DNN) in batches until all frames are exhausted. The second approach (Fig. 16(b)) uses a DNN with some type of recurrence, so that frames are processed in order. New frames are injected on the network and a corrected version of the image is obtained at the output. Introducing new frames on the input will slowly improve the quality of the output. This procedure can be iterated until a good enough final image is obtained. Both methods use the supervised learning where the training data is labeled by the corresponding MOMFBD result.

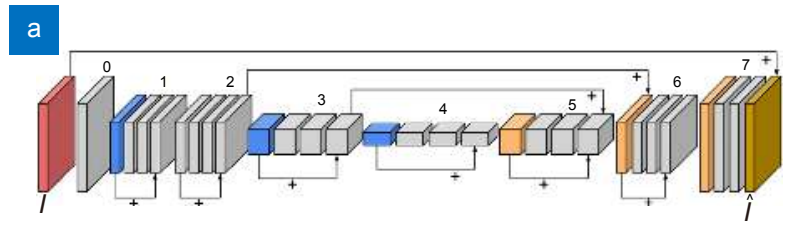

b
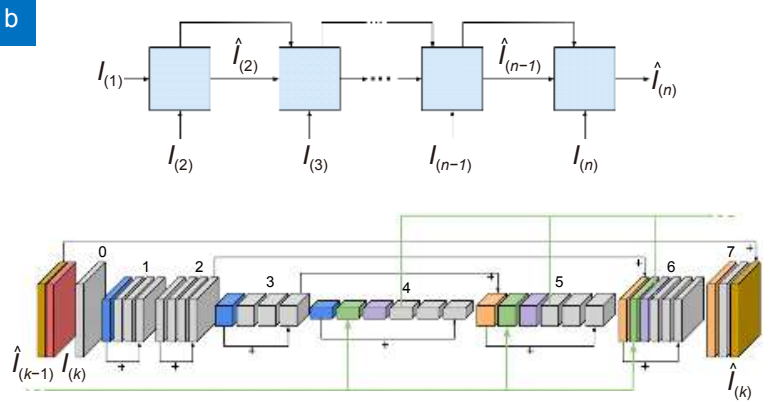

Fig. 16 | (a) Architecture of the encoder-decoder deconvolution neural network. The details of the architecture are described in Ref. ${ }^{95}$. (b) Upper panel: end-to-end deconvolution process, where the grey blocks are the deconvolution blocks described in the lower panel. Lower panel: internal architecture of each deconvolution block. Colors for the blocks and the specific details for each block are described in the reference. Figure reproduced from: $(a, b)$ Ref. ${ }^{95}$, ESO.

Figure 17 shows that both of the recurrent and encoder-decoder architectures are able to recover spatial periods between $\sim 3$ and $\sim 30$ pixels and increase their power, imitating what is done with MOMFBD. However, there is an apparent lack of sharpness and a slightly decrease of the power spectrums in the output of the networks compared with results of the MOMFBD in ref. ${ }^{95}$. The authors thought that part of the sharpness in the MOMFBD image was a consequence of the residual noise. The random selection of patches for building the training set has the desirable consequence of breaking a significant part of the spatial correlation of the noise in the MOMFBD images. Consequently, the networks are unable to reproduce it and, as a result, partially filter it out from the prediction. Thus, high photometric accuracy reconstructions can be obtained even when the images are degraded by noise. Furthermore, the architectures significantly accelerate the BD process and produce corrected images at a peak rate of $\sim 100$ images per second.

As both methods mentioned use the training data labeled by the corresponding MOMFBD result, the precision of the net depends on the photometric accuracy of the MOMFBD method. The supervised manner also limits its application in other areas. Besides, the loss functions are the $l_{2}$ distance between the deconvolved frames obtained at the output of the network and the one deconvolved with the MOMFBD algorithm. It is known that the $l_{2}$ norm of the residual tends to produce fuzzy reconstructions, especially when the number of frames is small. Those aspects can be improved in the future research of image reconstruction.

Another supervised manner deep learning architecture was proposed by Shi et al in $2019^{96}$. The authors proposed an end-to-end blind restoration method for ground-based space target images based on conditional generative adversarial network (cGAN) without estimating PSF. The flowchart of the model is displayed in Fig. 18. The training dataset of this network contains 4800 frames of simulated AO corrected space targets.

Figure 19 displays the reconstructed result of the Hubble telescope with cGAN. It could be seen that the quality of the restored image is improved. It not only accurately recovers the geometric contour of the image but also has remarkably improved some high-frequency details. The processing rate accelerates more than 100 times over traditional methods. Experimental results demonstrate that the proposed method not only enhances the restoration accuracy but also improves the restoration efficiency of single-frame object images at relative worse atmospheric conditions. Those improvements may be due to the improved loss function of this network. As no real space target images are used to validate the accuracy of the architecture, it is not clear about the robustness of this method.

The two methods above are based on the supervised manner and no information about the residual wavefront can be obtained in those procedures. In 2020, A. Asensio Ramos proposed an unsupervised method which can be trained simply with observations ${ }^{97}$. The block diagrams shown in Fig. 20 display the architecture of the 

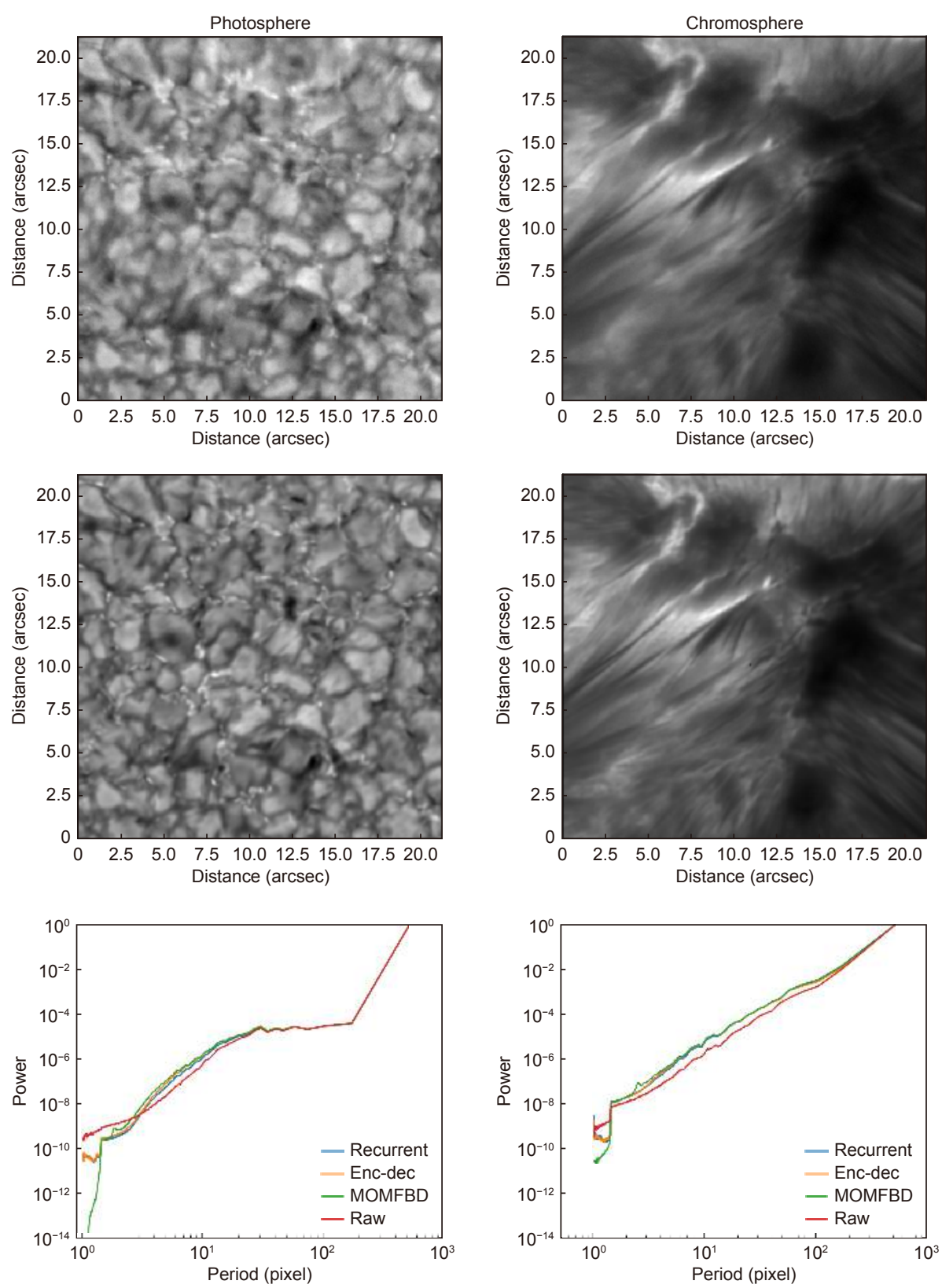

Fig. 17 | Top panels: a single raw image from the burst. Middle panels: reconstructed frames with the recurrent network. Lower panels: azimuthally averaged Fourier power spectra of the images. The left column shows results from the continuum image at $6302 \AA$ while the right column shows the results at the core of the $8542 \AA$ line. All power spectra have been normalized to the value at the largest period. Figure reproduced from ref. ${ }^{95}$, ESO.

network. The authors proposed a neural network model composed of three neural networks which are trained end-to-end. In the model, the linear image formation theory is introduced to construct a physically-motivated loss function. The analysis of the trained neural model shows that MFBD can be done by self-supervised training, i.e., using only observations. The outputs of the network are the corrected images and also estimations of the instantaneous wavefronts.

The training set consists of 26 bursts of 1000 images each with an exposure time of $30 \mathrm{~ms}$, enough to efficiently freeze the atmospheric turbulence. The images are taken at different times, and cover reasonably variable seeing conditions. Given the unsupervised character of the approach, the neural network can be easily refined by adding more observations which can cover different seeing conditions. Figure 21 displays the results of the method at the GJ661 object. The results of this operation are similar to the observed frames, apart from the obvious noise. It is obvious from the deconvolved 
images that the individual estimated wavefronts agree to some degree with the real ones.

The network model is on the order of 1000 times faster than applying standard deconvolution based on traditional optimization. With some work, the model can be used in real-time at the telescope. Given the lack of supervision, the method can be generally applied to any type of objects, once a sufficient amount of training data is available. Further improvement can be done by adding more training examples with a larger variety of objects, from point-like to extended ones.

The general information and differences of all the four networks described above are summarized in Table 7.

There are also some instructive researches on image deblurring and reconstruction including enhancing the SDO/HMI images using deep learning ${ }^{98}$, spatio-temporal filter adaptive network for video deblurring ${ }^{99}$ and the

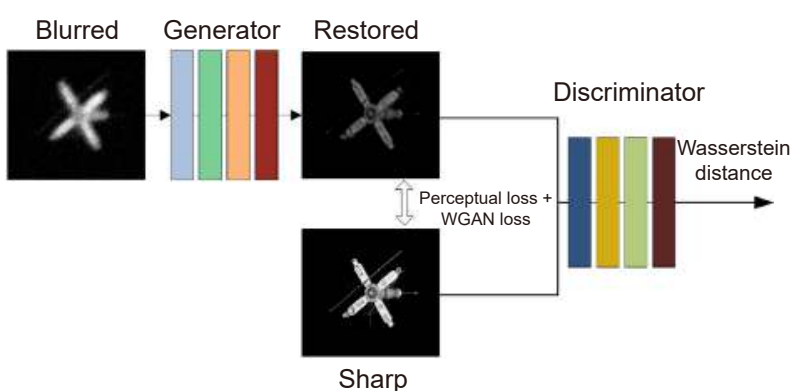

Fig. 18 | The flowchart of AO image restoration by cGAN. The whole network consists of two parts, generator network and discriminator network, which are used for learning the atmospheric degradation process and achieving the purpose of generating restored images. The loss function of the network is a combination of content loss for generator network and adversarial loss for discriminant network. Figure reproduced from ref. ${ }^{96}$, SPIE. deblurring of $\mathrm{AO}$ retinal images using deep $\mathrm{CNNs}^{100}$ etc. All the methods show wonderful outcomes at specific domain but the robustness of the architectures is doubtful. The accuracy is highly dependent on the independent identically distributed property of the training data and the test data. At the most time, the degraded procedures of the images observed by the ground-based telescopes are random and uncertain. That's why the deep learning reconstruction methods are not widely used by the astronomers until now. Training none end-to-end network and combining it with the imaging theory of the system may facilitate the applications of the machine learning in the post processing field.

\section{Others}

\section{Deep reinforcement learning for WFS-less AOS}

WFS-less AO is a type of AOS where no specific WFS is

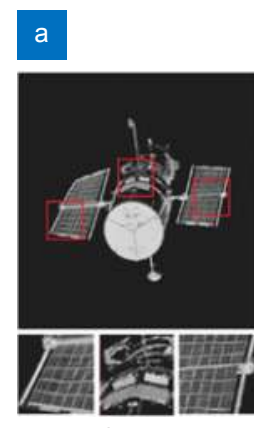

Sharp

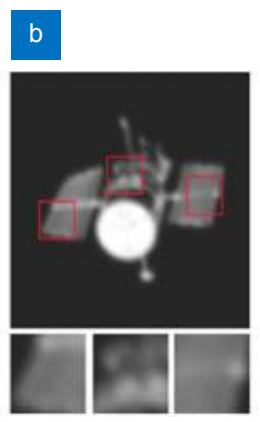

Blurred

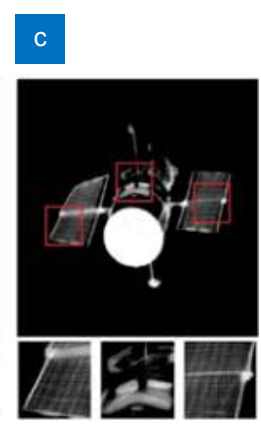

cGAN
Fig. 19 | The results of blind restoration for the Hubble telescope. (a) The sharp image, (b) the blurred image by Zernike polynomial method in atmospheric turbulence strength $D / r_{0}=10$, and (c) the result of restoration by $c G A N$, respectively. Figure reproduced from ref. ${ }^{96}$, SPIE.

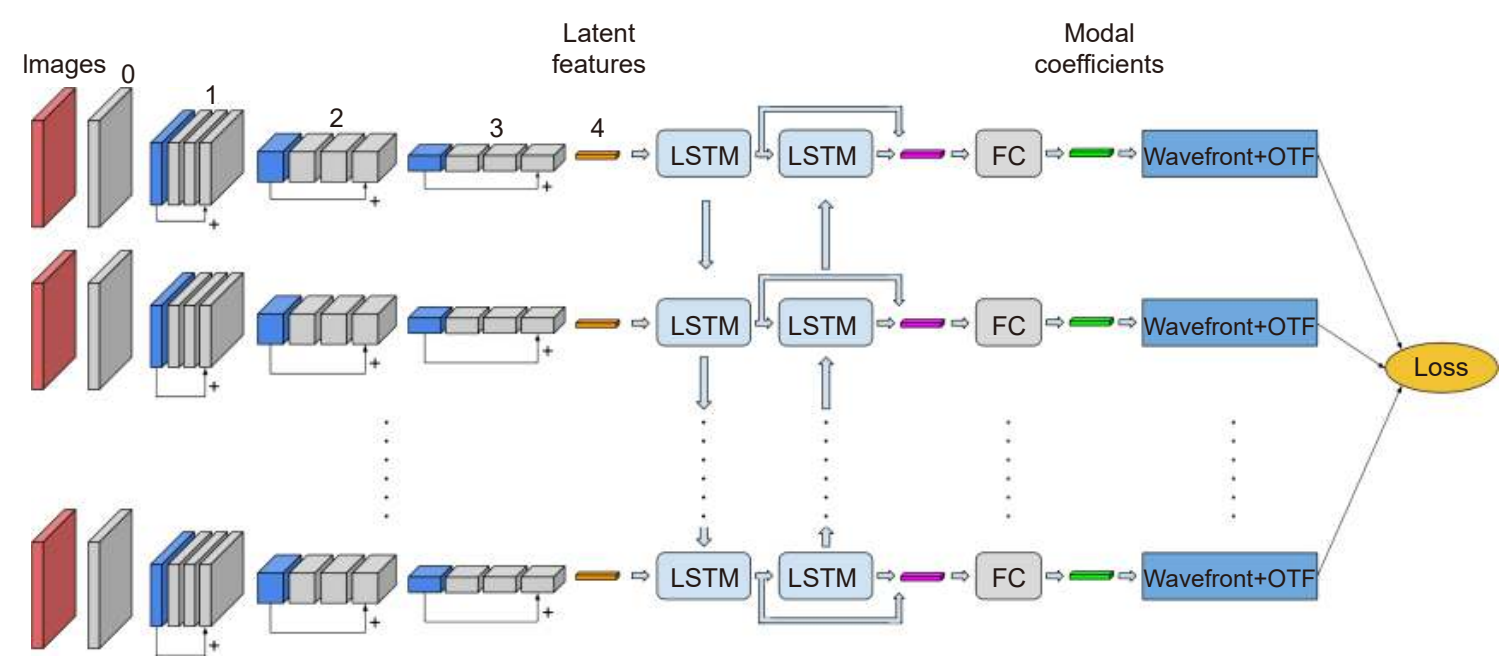

Fig. 20 | Block diagrams showing the architecture of the network and how it is trained by unsupervised training. Figure reproduced from ref. ${ }^{97}$, arXiv. 

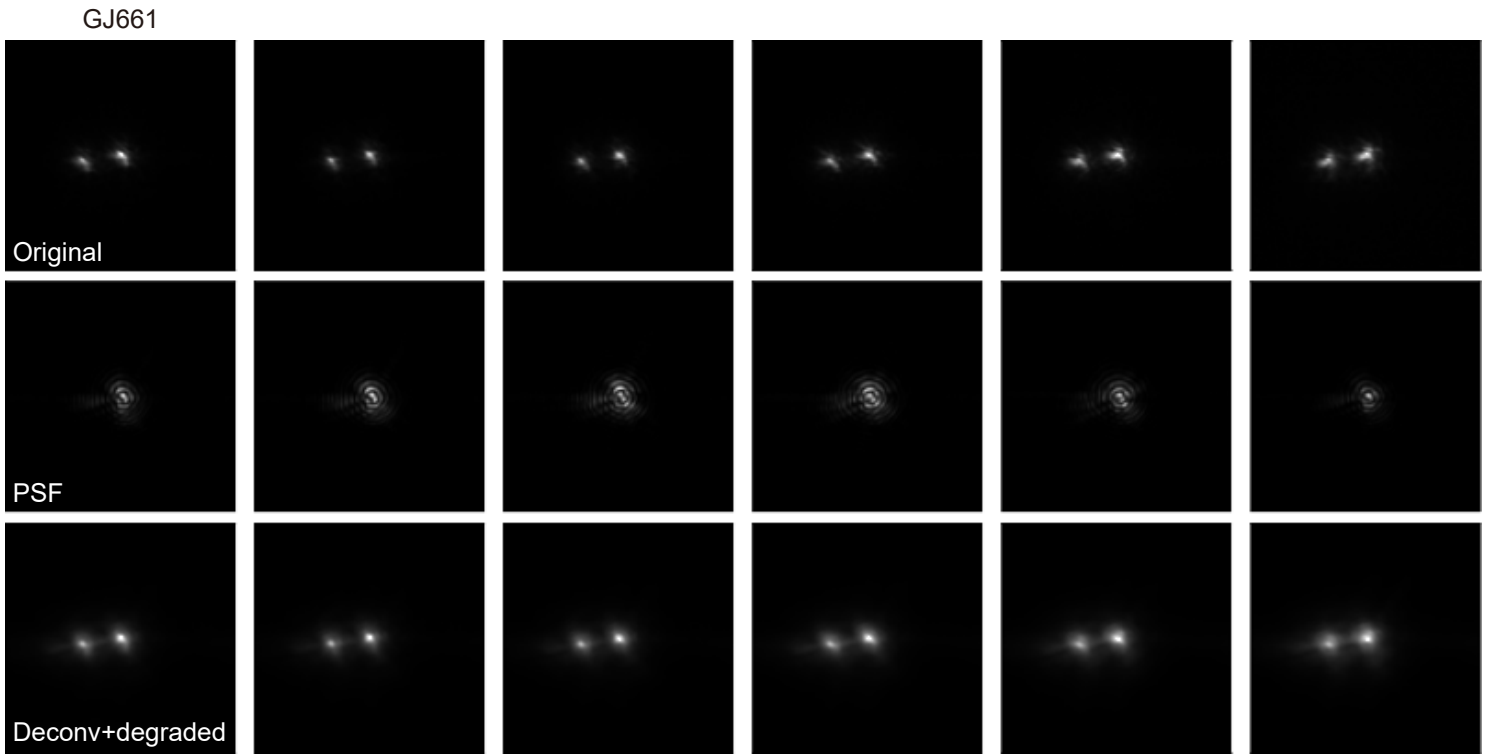

Fig. 21 | Original frames of the burst (upper row), estimated PSF (middle row) and for the GJ661. The upper row shows six raw frames of the burst. The second row displays the instantaneous PSF estimated by the neural network approach. The last row shows the results from reconvolve the deconvolved image with the estimated PSF. Figure reproduced from ref. ${ }^{97}$, arXiv.

Table 7 | The general information and differences of all the four networks.

\begin{tabular}{|c|c|c|c|c|c|}
\hline Neural network & Type & Training data & Label & Loss function & Output \\
\hline $\begin{array}{l}\text { Encoder-decoder } \\
\text { deconvolution }^{95}\end{array}$ & Supervised & $\begin{array}{c}80000 \text { patches of degraded } \\
\text { images }\end{array}$ & $\begin{array}{l}\text { MOMFBD } \\
\text { results }\end{array}$ & L2 metric & Deconvolved images \\
\hline $\begin{array}{l}\text { Recurrent encoder-decoder } \\
\text { deconvolution }{ }^{95}\end{array}$ & Supervised & $\begin{array}{c}80000 \text { patches of degraded } \\
\text { images }\end{array}$ & $\begin{array}{l}\text { MOMFBD } \\
\text { results }\end{array}$ & L2 metric & Deconvolved images \\
\hline $\begin{array}{c}\text { Conditional generative } \\
\text { adversarial }^{96}\end{array}$ & Supervised & $\begin{array}{c}4800 \text { frames of simulated } \\
\text { AO images }\end{array}$ & $\begin{array}{c}\text { Simulated } \\
\text { object }\end{array}$ & $\begin{array}{l}\text { Combination of content loss } \\
\text { and adversarial loss }\end{array}$ & Deconvolved images \\
\hline $\mathrm{CNN}^{-R N N^{97}}$ & Unsupervised & $\begin{array}{l}26000 \text { frames of observed } \\
\text { star images. }\end{array}$ & none & L2 metric & $\begin{array}{c}\text { Deconvolved images and } \\
\text { the wavefront }\end{array}$ \\
\hline
\end{tabular}

used. The WFS-less AO mainly has two approaches: model-free and model based. Model-free approach, such as $\mathrm{SPGD}^{101}$, is based on blind-optimization and its convergence speed is slow. The model-based approach is based on the approximately linear relation between the aberration features and the far-field intensity distribution features. Those features are designed by experts in the field. However, the linear relationship and feature optimality are often questionable. To solve the above problems, $\mathrm{Hu}$ proposed a self-learning control framework for WFS-less AOS through deep reinforcement learning ${ }^{102}$. The aberration correction process is expressed as a Markov decision process, which is represented by a 5 -tuple $(S, V, P, R, \gamma)$ : a state space $\mathrm{S}$, an action space $\mathrm{V}$, a state transition probability $P\left(S_{t+1} \mid S_{t}, V_{t}\right)$ : a reward function $R(s, v)$, and a discount factor $\gamma(\gamma \in[0,1])^{103}$. Compared with the model-free method, the deep learning method accelerates the convergence by the gained experience of value function. On the other hand, com- pared with the model-based method, a deep learning network extracts the features of the far field raw images and the deterministic policy gradient network can deal with the nonlinear relationship between extracted features.

\section{Machine learning for AO modelling}

In order to cope with the disturbance of slope response matrix and improve the adaptive ability of AO control system, $\mathrm{Xu}$ proposed a deep learning control model (DLCM) ${ }^{104}$. The PI and the DLCM control models are shown in Fig. 22.

The DLCM consists of a model net and an actor network. The model network and actor network have the same structure but have different roles. The model network shares the trained parameters with the actor network to stabilize the output of the actor network and improve the convergence speed of the actor network. The actor network updates the decision sample space and guides the update of the model network. Furthermore, 

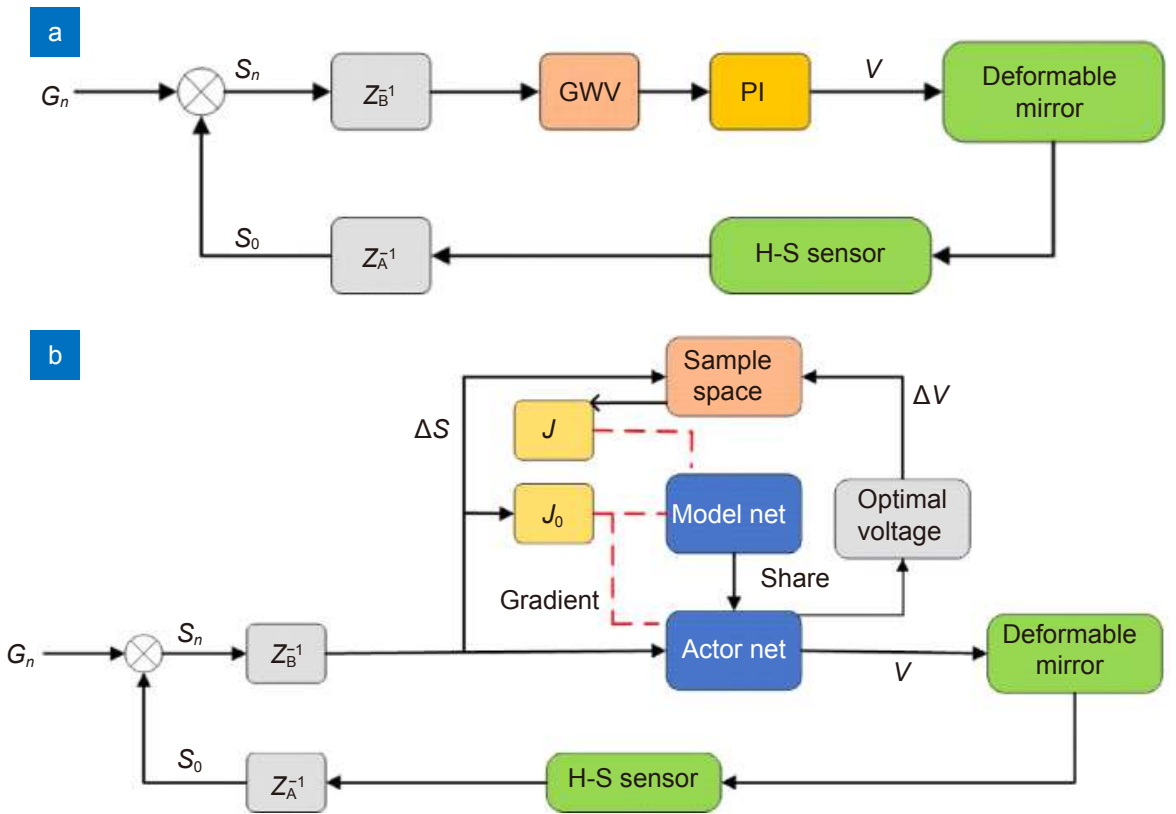

Fig. 22 | Controller for an AOS. (a) PI control model; (b) DLCM control model. $Z_{A}^{-1}$ and $Z_{B}^{-1}$ are the H-S sensor delay and control calculation delay, respectively. GWV (Generating wavefront to voltage). $J$ and $J_{0}$ are the cost functions of the network and the red dotted line is a gradient data stream. Figure reproduced from ref. ${ }^{104}$, Optical Society of America.

compared to the fixed parameter PI control, real-time update of the actor network can cope with the disturbance of slope response matrix and improve control accuracy of the DLCM.

\section{Conclusion \& discussion}

Recent advances in IAO are summarized including the intelligent wavefront sensing, wavefront reconstruction and control as well as post-processing. By using the machine learning, a lot of inverse or complex problems can be solved if large-scale datasets are available. Two main scenes seem to be particularly suitable to use machine learning at present. One is to use the ANN to build the relationship between the measurement (image) and the wavefront for wavefront reconstruction or the relationship between blurred image and the diffraction-limited image for post-processing. On one hand, features extracted from data may perform better than the manually selected linear ones. On the other hand, some iterative methods for phase retrieval may be replaced by deep learning for faster speed. The other is to use the ANN to do the nonlinear wavefront prediction for better accuracy and more importantly to adapt to the non-stationary turbulence.

Besides of the algorithms, the large-scale and highquality data is also important which may be not easy to get. Some data can be generated by the computer for the training but its adaptability to the real systems needs to be demonstrated. Although lots of simulation and laboratory results have been obtained, less have been used in real AOSs recently. The application of IAO in real system is of great importance for demonstrating the generalization ability and real-time performance. As long as an on-sky demonstrator succeeds, lots of great progresses can be expected. In the more distant future, with the development of unsupervised learning and reinforcement learning, we can imagine an IAO system that can keep learning the rules from on-line multisource data to improve itself.

\section{References}

1. Rao $\mathrm{CH}$, Zhu L, Rao XJ, Zhang LQ, Bao H et al. Instrument description and performance evaluation of a high-order adaptive optics system for the $1 \mathrm{~m}$ new vacuum solar telescope at Fuxian solar observatory. Astrophys J 833, 210 (2016).

2. Kim D, Choi H, Brendel $\mathrm{T}$ et al. Advances in optical engineering for future telescopes. Opto-Electron Adv 4 (2021).

3. Tyson RK. Adaptive optics and ground-to-space laser communications. Appl Opt 35, 3640-3646 (1996).

4. Kubby JA. Adaptive Optics for Biological Imaging (Taylor \& Francis, Boca Raton, America, 2013).

5. Shi GH, Dai Y, Wang L, Ding ZH, Rao XJ et al. Adaptive optics optical coherence tomography for retina imaging. Chin Opt Lett 6, 424-425 (2008).

6. Booth MJ. Adaptive optical microscopy: the ongoing quest for a perfect image. Light Sci App/ 3, e165 (2014).

7. Bennet F, Thearle O, Roberts L, Smith J, Spollard SJ et al. Free-space quantum communication link with adaptive optics. (AMOS, 2018). 
8. https://afresearchlab.com/news/afrl-demonstrates-worlds-firstdaytime-free-space-quantum-communication-enabled-by-adaptive-optics/

9. Burns SA, Elsner AE, Sapoznik KA, Warner RL, Gast TJ. Adaptive optics imaging of the human retina. Prog Retin Eye Res 68, 1-30 (2019).

10. Jiang $\mathrm{WH}$. Overview of adaptive optics development. OptoElectron Eng 45, 170489 (2018).

11. Rao $\mathrm{CH}$, Zhu L, Zhang LQ et al. Development of solar adaptive optics. Opto-Electron Eng 45, 170733 (2018).

12. Wei $\mathrm{K}$, Zhang $\mathrm{XJ}$, Xian $\mathrm{H}$, Ma WL, Zhang $A$ et al. First light on the 127-element adaptive optical system for 1.8-m telescope. Chin Opt Lett 8, 1019-1021 (2010).

13. Yang ZP, Li ED, Zhang XJ, Fan MW, Shi NP et al. Adaptive optics correction systems on Shen Guang III facility. Opto-Electron Eng 45, 180049 (2018)

14. Liu C, Chen SQ, Li XY, Xian H. Performance evaluation of adaptive optics for atmospheric coherent laser communications. Opt Express 22, 15554-15563 (2014).

15. Zhao JL, Xiao F, Kang J, Zhao HX, Dai $Y$ et al. Quantifying intraocular scatter with near diffraction-limited double-pass point spread function. Biomed Opt Express 7, 4595-4604 (2016).

16. Guo YM, Zhang A, Fan XL, Rao $\mathrm{CH}$, Wei $\mathrm{L}$ et al. First on-sky demonstration of the piezoelectric adaptive secondary mirror. Opt Lett 41, 5712-5715 (2016).

17. Rao $\mathrm{CH}$, Zhang LQ, Kong L, Guo YM, Rao XJ et al. First light of solar multi-conjugate adaptive optics at the 1-m new vacuum solar telescope. Sci China Phys, Mech Astron 61, 089621 (2018).

18. Rao CH, Gu NT, Rao XJ, Li C, Zhang LQ et al. First light of the 1.8-m solar telescope-CLST. Sci China Phys, Mech Astron 63, 109631 (2020).

19. Chen M, Liu C, Rui DM, Xian H. Experimental results of 5-Gbps free-space coherent optical communications with adaptive optics. Opt Commun 418, 115-119 (2018).

20. Guan CL, Zhang XJ, Deng JM et al. Deformable mirror technologies at Institute of Optics and Electronics, Chinese Academy of Sciences. Opto-Electron Eng 47, 200337 (2020).

21. Huang $\mathrm{LH}$, Rao $\mathrm{CH}$. Wavefront sensorless adaptive optics: a general model-based approach. Opt Express 19, 317-379 (2011).

22. Assémat F, Gendron E, Hammer F. The FALCON concept: multi-object adaptive optics and atmospheric tomography for integral field spectroscopy - principles and performance on an 8m telescope. Mon Not Roy Astron Soc 376, 287-312 (2007).

23. Macintosh B, Troy M, Doyon R, Graham J, Baker K et al. Extreme adaptive optics for the Thirty Meter Telescope. Proc SPIE 6272, 62720N (2006).

24. LeCun $\mathrm{Y}$, Bengio $\mathrm{Y}$, Hinton G. Deep learning. Nature 512, 436-444 (2015).

25. Angel JRP, Wizinowich P, Lloyd-Hart M, Sandler D. Adaptive optics for array telescopes using neural-network techniques. Nature 348, 221-224 (1990).

26. Sandler DG, Barrett TK, Palmer DA, Fugate FQ, Wild WJ. Use of a neural network to control an adaptive optics system for an astronomical telescope. Nature 351, 300-302 (1991).

27. Demailly L, Gendron E, Beuzit JL, Lacombe F, Hubin NN. Artificial Intelligence system and optimized modal control for the ADONIS adaptive optics instrument. Proc SPIE 2201, 867-878 (1994).
28. Wizinowich PL, Lloyd-Hart M, McLeod BA, Colucci D, Dekany $\mathrm{RG}$ et al. Neural network adaptive optics for the multiple-mirror telescope. Proc SPIE 1542, 148-158 (1991).

29. Lloyd-Hart M, Wizinowich P, McLeod B, Wittman D, Colucci D et al. First results of an on-line adaptive optics system with atmospheric wavefront sensing by an artificial neural network. Astrophys J 390, L41-L44 (1992).

30. Barrett TK, Sandler DG. Artificial neural network for the determination of Hubble Space Telescope aberration from stellar images. Appl Opt 32, 1720-1727 (1993).

31. Montera DA, Welsh BM, Roggemann MC, Ruck DW. Prediction of wave-front sensor slope measurements with artificial neural networks. Appl Opt 36, 675-681 (1997).

32. Davies R, Kasper M. Adaptive optics for astronomy. Ann Rev Astron Astrophys 50, 305-351 (2012).

33. Platt BC, Shack R. History and principles of Shack-Hartmann wavefront sensing. J Refract Surg 17, S573-S577 (2001).

34. Ragazzoni R. Pupil plane wavefront sensing with an oscillating prism. J Mod Opt 43, 289-293 (1996).

35. Roddier F. Curvature sensing and compensation: a new concept in adaptive optics. App/ Opt 27, 1223-1225 (1988).

36. Noll RJ. Zernike polynomials and atmospheric turbulence. J Opt Soc Am 66, 207-211 (1976).

37. Dai GM. Modal wave-front reconstruction with Zernike polynomials and Karhunen-Loève functions. J Opt Soc Am A 13, 1218-1225 (1996).

38. Boyer C, Michau V, Rousset G. Adaptive optics: Interaction matrix measurements and real time control algorithms for the COME-ON project. Proc SPIE 1271, 63-81 (1990).

39. Kulcsár C, Raynaud HF, Petit C, Conan JM, de Lesegno PV. Optimal control, observers and integrators in adaptive optics. Opt Express 14, 7464-7476 (2006).

40. Petit C, Quiros-Pacheco F, Conan JM, Kulcsar C, Raynaud HF et al. Kalman-filter-based control for adaptive optics. Proc SPIE 5490, 1414-1425 (2004).

41. Petit C, Conan JM, Kulcsár C, Raynaud HF, Fusco T et al. First laboratory demonstration of closed-loop Kalman based optimal control for vibration filtering and simplified MCAO. Proc SPIE 6272, 62721T (2006).

42. Petit C, Conan JM, Kulcsár C, Raynaud HF, Fusco T. First laboratory validation of vibration filtering with LQG control law for Adaptive Optics. Opt Express 16, 87-97 (2008).

43. Correia C, Conan JM, Kulcsár C, Raynaud HF, Petit C. Adapting optimal LQG methods to ELT-sized AO systems. In Proceedings of the 1st AO4ELT Conference 07003 (EDP Sciences, 2010); https://doi.org/10.1051/ao4elt/201007003.

44. Wang JY, Guo YM, Kong L, Rao CH. Experimental demonstration of LQG control with disturbance mitigation on multiple modes in adaptive optics system. Optik 202, 163594 (2020).

45. Wang JY, Guo YM, Kong L, Zhao LQ, Gu NT et al. Automatic disturbance identification for linear quadratic Gaussian control in adaptive optics. Mon Not Roy Astronom Soc 496, 5126-5138 (2020).

46. Doelman N, Osborn J. Modelling and prediction of non-stationary optical turbulence behavior. Proc SPIE 9909, 99091M (2016).

47. Ayers GR, Dainty JC. Iterative blind deconvolution method and its applications. Opt Lett 13, 547-549 (1988).

48. $\mathrm{Ng}$ MK, Plemmons RJ, Qiao SZ. Regularization of RIF blind image deconvolution. IEEE Trans Image Process $\mathbf{9}$, 
1130-1134 (2000).

49. Thiébaut E, Conan JM. Strict a priori constraints for maximumlikelihood blind deconvolution. J Opt Soc Am A12, 485-492 (1995).

50. Paxman RG, Schulz TJ, Fienup JR. Joint estimation of object and aberrations by using phase diversity. J Opt Soc Am A $\mathbf{9}$, 1072-1085 (1992).

51. Löfdahl MG, Scharmer GB. Wavefront sensing and image restoration from focused and defocused solar images. Astron Astrophys Supp/ Ser 107, 243-264 (1994).

52. Löfdahl MG, Berger TE, Shine RS, Title AM. Preparation of a dual wavelength sequence of high-resolution solar photospheric images using phase diversity. Astrophys $J$ 495, 965-972 (1998).

53. Schulz TJ, Stribling BE, Miller JJ. Multiframe blind deconvolution with real data: imagery of the Hubble Space Telescope. Opt Express 11, 355-362 (1997).

54. Schulz TJ. Multiframe blind deconvolution of astronomical images. J Opt Soc Am A 10, 1064-1073 (1993).

55. Löfdahl MG. Multi-frame blind deconvolution with linear equality constraints. Proc SPIE 4792, 146-155 (2002)

56. Van Noort M, Van Der Voort R, Löfdahl MG. Solar image restoration by use of multi-frame blind de-convolution with multiple objects and phase diversity. Sol Phys 228, 191-215 (2005).

57. Wöger $F$, van der Lühe $O$. Field dependent amplitude calibration of adaptive optics supported solar speckle imaging. Appl Opt 46, 8015-8026 (2007).

58. Wöger F. Optical transfer functions derived from solar adaptive optics system data. Appl Opt 49, 1818-1825 (2010).

59. Labeyrie A. Attainment of diffraction limited resolution in large telescopes by Fourier analysing speckle patterns in star images. Astron Astrophys 6, 85-87 (1970).

60. Weigelt GP. Modified astronomical speckle interferometry "speckle masking". Opt Commun 21, 55-59 (1977).

61. Pehlemann E, van der Lühe O. Technical aspects of the speckle masking phase reconstruction algorithm. Astron Astrophys 216, 337-346 (1989).

62. Puschmann KG, Sailer M. Speckle reconstruction of photometric data observed with adaptive optics. Astron Astrophys 454, 1011-1019 (2006).

63. Knox KT, Thompson BJ. Recovery of images from atmospherically degraded short-exposure photographs. Astron Astrophys 193, L45-L48 (1974).

64. van Noort M, van der Voort LR, Löfdahl MG. Solar image restoration by use of multi-object multi-frame blind deconvolution. In Solar MHD Theory and Observations: A High Spatial Resolution Perspective ASP Conference Series (ASP, 2006); https://ui.adsabs.harvard.edu/abs/2006ASPC..354...55V.

65. Puschmann KG, Beck C. Application of speckle and (multi-object) multi-frame blind deconvolution techniques on imaging and imaging spectropolarimetric data. Astron Astrophys 533, A21 (2011).

66. Peck CL, Wöger F, Marino J. Influence of speckle image reconstruction on photometric precision for large solar telescopes. Astron Astrophys 607, A83 (2017).

67. Zhong LB, Zhang LQ, Shi ZD, Tian Y, Guo YM et al. Wide fieldof-view, high-resolution Solar observation in combination with ground layer adaptive optics and speckle imaging. Astron Astrophys 637, A99 (2020)

68. Denker C, Yang G, Wang H. Near real-time image reconstruc- tion. Solar Phys 202, 63-70 (2001).

69. Wöger F, van der Lühe O. KISIP: a software package for speckle interferometry of adaptive optics corrected solar data. Proc SPIE 7019, 7019E (2008).

70. Guo T, Zhang B, Gu N T et al. Research on polarization Hartmann wavefront detection technology. Opto-Electron Eng 48, 210076 (2021).

71. Guo H, Korablinova N, Ren QS, Bille J. Wavefront reconstruction with artificial neural networks. Opt Express 14, 6456-6462 (2006).

72. Swanson R, Lamb M, Correia C, Sivanandam S, Kutulakos K. Wavefront reconstruction and prediction with convolutional neural networks. Proc SPIE 10703, 107031F (2018).

73. Li ZQ, Li XY. Centroid computation for Shack-Hartmann wavefront sensor in extreme situations based on artificial neural networks. Opt Express 26, 31675-31692 (2018).

74. Gómez SLS, González-Gutiérrez C, Alonso ED, Rodríguez JDS, Rodríguez MLS et al. Improving adaptive optics reconstructions with a deep learning approach. In Proceedings of the 13th International Conference on Hybrid Artificial Intelligence Systems (Springer, 2018);https://doi.org/10.1007/978-3-31992639-1_7.

75. DuBose TB, Gardner DF, Watnik AT. Intensity-enhanced deep network wavefront reconstruction in Shack-Hartmann sensors. Opt Lett 45, 1699-1702 (2020).

76. Hu LJ, Hu SW, Gong W, Si K. Learning-based Shack-Hartmann wavefront sensor for high-order aberration detection. Opt Express 27, 33504-33517 (2019).

77. Hu LJ, Hu SW, Gong W, Si K. Deep learning assisted ShackHartmann wavefront sensor for direct wavefront detection. Opt Lett 45, 3741-3744 (2020).

78. Kendrick RL, Acton DS, Duncan AL. Phase-diversity wave-front sensor for imaging systems. Appl Opt 33, 6533-6546 (1994).

79. Paine SW, Fienup JR. Machine learning for improved imagebased wavefront sensing. Opt Lett 43, 1235-1238 (2018).

80. Guo HY, Xu YJ, Li Q, Du SP, He D et al. Improved machine learning approach for wavefront sensing. Sensors 19, 3533 (2019).

81. Ma HM, Liu HQ, Qiao Y, Li XH, Zhang W. Numerical study of adaptive optics compensation based on Convolutional Neural Networks. Opt Commun 433, 283-289 (2019).

82. Wu Y, Guo YM, Bao H, Rao CH. Sub-millisecond phase retrieval for phase-diversity wavefront sensor. Sensors 20, 4877 (2020).

83. Nishizaki $Y$, Valdivia M, Horisaki $R$, Kitaguchi $K$, Saito $M$ et al. Deep learning wavefront sensing. Opt Express 27, 240-251 (2019).

84. Osborn J, Guzman D, de Cos Juez FJ, Basden AG, Morris TJ et al. Open-loop tomography with artificial neural networks on CANARY: on-sky results. Mon Not Roy Astron Soc 441, 2508-2514 (2014).

85. Jorgenson MB, Aitken GJM. Prediction of atmospherically induced wave-front degradations. Opt Lett 17, 466-468 (1992).

86. Lloyd-Hart M, McGuire P. Spatio-temporal prediction for adaptive optics wavefront reconstructors. In Proceedings of the European Southern Observatory Conference on Adaptive Optics (ESO, 1996); http://citeseerx.ist.psu.edu/viewdoc/download? doi=10.1.1.35.3278\&rep=rep1\&type=pdf.

87. van Kooten M, Doelman N, Kenworthy M. Impact of time-variant turbulence behavior on prediction for adaptive optics systems. 
J Opt Soc Am A 36, 731-740 (2019).

88. Doelman N, Fraanje R, Houtzager I, Verhaegen M. Adaptive and real-time optimal control for adaptive optics systems. Eur $J$ Control 15, 480-488 (2009).

89. Chen Y. LSTM recurrent neural network prediction algorithm based on Zernike modal coefficients. Optik 203, 163796 (2020).

90. Chen Y. Voltages prediction algorithm based on LSTM recurrent neural network. Optik 220, 164869 (2020).

91. Liu XW, Morris T, Saunter C, de Cos Juez FJ, GonzálezGutiérrez $\mathrm{C}$ et al. Wavefront prediction using artificial neural networks for open-loop adaptive optics. Mon Not Roy Astron Soc 496, 456-464 (2020).

92. Sun Z, Chen Y, Li XY, Qin XL, Wang HY. A Bayesian regularized artificial neural network for adaptive optics forecasting. Opt Commun 382, 519-527 (2017).

93. van Kooten M, Doelman N, Kenworthy M. Performance of AO predictive control in the presence of non-stationary turbulence. In Proceedings of the 5th AO4ELT Conference (Instituto de Astrofisica de Canarias, 2017);

https://repository.tudelft.nl/view/tno/uuid\%3A4a101b83-7e9044f8-abaa-b7817cc8b16a.

94. Béchet $\mathrm{C}$, Tallon M, Le Louarn M. Very low flux adaptive optics using spatial and temporal priors. In Proceedings of the 1st AO4ELT Conference 03010 (EDP Sciences, 2010); https://doi.org/10.1051/ao4elt/201003010

95. Ramos AA, de la Cruz Rodríguez J, Yabar AP. Real-time, multiframe, blind deconvolution of solar images. Astron Astrophys 620, A73 (2018).

96. Shi JL, Zhang RZ, Guo SP, Yang YK, Xu R et al. Space targets adaptive optics images blind restoration by convolutional neural network. Opt Eng 58, 093102 (2019).

97. Ramos AA. Learning to do multiframe blind deconvolution unsupervisedly. arXiv: 2006.01438 (2020). https://doi.org/10.48550/arXiv.2006.01438

98. Baso CJD, Ramos AA. Enhancing SDO/HMI images using deep learning. Astron Astrophys 614, A5 (2018).

99. Zhou SC, Zhang JW, Pan JS, Zuo WM, Xie HZ et al. Spatiotemporal filter adaptive network for video deblurring. In Pro- ceedings of 2019 IEEE/CVF International Conference on Computer Vision (IEEE, 2019);

https://doi.org/10.1109/ICCV.2019.00257.

100. Xiao F, Zhao JL, Zhao HX, Yun D, Zhang YD. Deblurring adaptive optics retinal images using deep convolutional neural networks. Biomed Opt Express 8, 5675-5687 (2017).

101. Vorontsov MA, Sivokon VP. Stochastic parallel-gradient-descent technique for high-resolution wave-front phase-distortion correction. J Opt Soc Am A 15, 2745-2758 (1998).

102. Hu K, Xu B, Xu ZX, Wen LH, Yang P et al. Self-learning control for wavefront sensorless adaptive optics system through deep reinforcement learning. Optik 178, 785-793 (2019).

103. Mnih V, Kavukcuoglu K, Silver D, Graves A, Antonoglou I et al. Playing Atari with deep reinforcement learning. arXiv: 1312.5602 (2013). https://doi.org/10.48550/arXiv.1312.5602.

104. Xu ZX, Yang P, Hu K, Xu B, Li HP. Deep learning control model for adaptive optics systems. Appl Opt 58, 1998-2009 (2019).

\section{Acknowledgements}

This work was funded by the National Natural Science Foundation of China (12173041, 11733005, 11727805), Youth Innovation Promotion Association, Chinese Academy of Sciences (No. 2020376), Frontier Research Fund of Institute of Optics and Electronics, Chinese Academy of Sciences (No. C21K002), Research Equipment Development Project of the Chinese Academy of Sciences (No. YA18K019) and Laboratory Innovation Foundation of the Chinese Academy of Sciences (No. YJ20K002).

\section{Author contributions}

Y. M. Guo prepared the part of introduction, wavefront control and the first draft of the paper. L. B. Zhong prepared the part of post-processing. L. Min prepared the part of machine learning for WFS-Less. J. Y. Wang prepared for the part of traditional wavefront control. Y. Wu prepared the part of wavefront sensing.

All authors commented on the manuscript.

\section{Competing interests}

The authors declare no competing financial interests. 